\title{
2-10 keV luminosity of high-mass binaries as a gauge of ongoing star-formation rate ${ }^{\star}$
}

\author{
M. Persic ${ }^{1}$, Y. Rephaeli²,3, V. Braito ${ }^{4}$, M. Cappi $^{5}$, R. Della Ceca ${ }^{4}$, A. Franceschini ${ }^{6}$, and D. E. Gruber ${ }^{7}$ \\ 1 INAF/Osservatorio Astronomico di Trieste, via G.B. Tiepolo 11, 34131 Trieste, Italy \\ 2 School of Physics and Astronomy, Tel Aviv University, Tel Aviv 69978, Israel \\ 3 CASS, University of California, San Diego, La Jolla, CA 92093, USA \\ ${ }^{4}$ INAF/Osservatorio Astronomico di Brera, via Brera 28, 20121 Milano, Italy \\ 5 INAF/IASF, via P. Gobetti 101, 40129 Bologna, Italy \\ ${ }^{6}$ Dipartimento di Astronomia, Università di Padova, vicolo Osservatorio 2, 35122 Padova, Italy \\ 74789 Panorama Drive, San Diego, CA 92116, USA
}

Received 13 October 2003 / Accepted 7 February 2004

\begin{abstract}
Based on recent work on spectral decomposition of the emission of star-forming galaxies, we assess whether the integrated 2-10 keV emission from high-mass X-ray binaries (HMXBs), $L_{2-10}^{\mathrm{HMXB}}$, can be used as a reliable estimator of ongoing star formation rate $(S F R)$. Using a sample of 46 local $(z \lesssim 0.1)$ star-forming galaxies, and spectral modeling of ASCA, BeppoSAX, and $X M M$-Newton data, we demonstrate the existence of a linear $S F R-L_{2-10}^{\mathrm{HMXB}}$ relation which holds over $\sim 5$ decades in X-ray luminosity and $S F R$. The total $2-10 \mathrm{keV}$ luminosity is not a precise $S F R$ indicator because at low $S F R$ (i.e., in normal and moderately-starbursting galaxies) it is substantially affected by the emission of low-mass X-ray binaries, which do not trace the current $S F R$ due to their long evolution lifetimes, while at very high SFR (i.e., for very luminous FIR-selected galaxies) it is frequently affected by the presence of strongly obscured AGNs. The availability of purely SB-powered galaxies - whose $2-10 \mathrm{keV}$ emission is mainly due to HMXBs - allows us to properly calibrate the $S F R-L_{2-10}^{\mathrm{HMXB}}$ relation. The $S F R-L_{2-10}^{\mathrm{HMXB}}$ relation holds also for distant $(z \sim 1)$ galaxies in the Hubble Deep Field North sample, for which we lack spectral information, but whose $S F R$ can be estimated from deep radio data. If confirmed by more detailed observations, it may be possible to use the deduced relation to identify distant galaxies that are X-ray overluminous for their (independently estimated) $S F R$, and are therefore likely to hide strongly absorbed AGNs.
\end{abstract}

Key words. X-rays: galaxies - galaxies: spiral - galaxies: starburst - X-rays: binaries - infrared: galaxies radio continuum: galaxies

\section{Introduction}

X-ray emission of star-forming galaxies (SFGs) consists of various components including discrete sources, such as X-ray binaries and supernova remnants (SNRs), diffuse hot gas, Compton scattering of ambient FIR photons, and possibly an active nucleus. The resulting integrated spectra harbor the signatures of these emission components.

Persic \& Rephaeli (2002, hereafter PR02) have quantitatively assessed the roles of the various X-ray emission mechanisms in SFGs. They have used an equilibrium stellarpopulation synthesis model of the Galactic population of X-ray binaries (Iben et al. 1995a,b) to deduce birthrates for interacting binaries; these, combined with estimates of the duration of the X-ray bright phase, have allowed PR02 to make realistic estimates of the relative (Galactic) abundances of high-mass and low-mass X-ray binaries (HMXBs, LMXBs). The abundance

\footnotetext{
Send offprint requests to: M. Persic, e-mail: persic@ts . astro.it

$\star$ Appendix A is only available in electronic form at http://www. edpsciences.org
}

of SNRs (both type II and Ia explosions) was also consistently estimated. From the literature PR02 derived typical spectra for these classes of source. The spectral properties and relative abundances of the various classes of stellar sources determine the composite X-ray spectrum arising from a stellar population of Galactic composition. Therefore, the PR02 "stellar" contribution has then no essential degrees of freedom: fixed by the synthetic model of the Galactic population of binaries and by the observed X-ray spectra of the contributing components, it represents the X-ray spectrum emitted by a Galactic mix of HMXBs and LMXBs. As such, the stellar component of the PR02 template spectrum is likely to be appropriate for a quietly star forming galaxy like the Milky Way (see Fig. 1-left).

${ }^{1}$ The diffuse non-stellar part of the PR02 synthetic spectrum has both a thermal and a non-thermal component. The former is related to the SN-powered outgoing galactic wind and is mostly relevant at energies $\lesssim 1 \mathrm{keV}$. The latter is due to Compton scattering of the $\mathrm{SN}$-accelerated, radio-emitting relativistic electrons off the FIR and CMB radiation fields, and it arguably dominates the spectrum of SFGs at energies of $\gtrsim 30 \mathrm{keV}$ (see Persic \& Rephaeli 2002, 2003). 

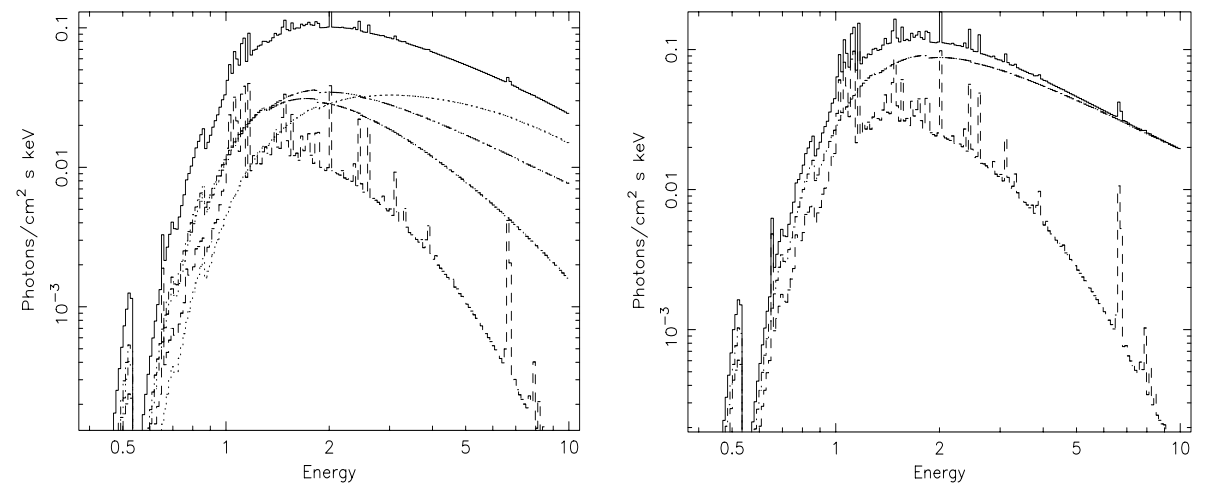

Fig. 1. The integrated "stellar" emission in the template X-ray spectrum of a star-forming galaxy proposed by Persic \& Rephaeli (2002). Two different cases are shown: "standard" (left) and "top-heavy" (right). Shown for the standard case in ascending order at 7 keV: SNRs, faint LMXBs, HMXBs, and bright LMXBs. On the right, in ascending order at $3 \mathrm{keV}$, only SNRs and HMXBs are shown. The assumed 0.5-50 keV luminosities and number abundances are: $\log L_{\mathrm{x}}=37.7$ for HMXBs (50 objects) and high-luminosity LMXBs (70 objects), 37.0 for SNRs (20 objects), and 36.7 for low-luminosity LMXBs (130 objects). The spectral components are normalized in energy flux in the $0.5-50 \mathrm{keV}$ band. The spectrum is absorbed through a HI column density $N_{\mathrm{H}}=10^{22} \mathrm{~cm}^{-2}$.

The PR02 approach has the flexibility of handling also the extreme cases of "no ongoing SF" and "very high ongoing SF" by switching off the HMXB and SNR components and, respectively, the LMXB component (see Fig.1-right). By letting the amplitudes of the various spectral components vary (while keeping their profiles fixed) in the spectral fit, one can use the PR02 procedure to determine the SF state of a given galaxy. Thus, this treatment is generally valid and not limited to any particular regime of SF activity.

Based on their survey of galactic X-ray emission mechanisms, PR02 concluded that in the $2-10 \mathrm{keV}$ energy range $\mathrm{X}$-ray binaries of both types, HMXBs and LMXBs, are the most prominent components with the required spectral shapes (see Fig. 1-left). These have either a power-law (PL) form for HMXBs, or cut-off PL for LMXBs, with observed ranges of spectral parameters (HMXBs: $\Gamma \simeq 1.0-1.4$, see White et al. 1983; LMXBs: $\Gamma \simeq 0-1.4, E_{\mathrm{c}} \sim 5-10 \mathrm{keV}$, see Christian \& Swank 1997) that provide good fits to the spatially unresolved BeppoSAX spectra of the most extensively observed nearby starburst galaxies (SBGs), M 82 and NGC 253 (see PR02), and to the XMM-Newton spectra of a number of distant UltraLuminous Infra-Red Galaxies (ULIRGs; Franceschini et al. 2003).

From a broader perspective, the template spectrum of PR02 provides a framework that may prove especially useful for interpreting low spatial resolution data on SFGs, either distant (from Chandra and XMM: e.g., Hornschemeier et al. 2001, 2003; Alexander et al. 2002; Bauer et al. 2002; Franceschini et al. 2003) or nearby (from ASCA and BeppoSAX: e.g., Cappi et al. 1999; Dahlem et al. 1998; Della Ceca 1996, 1997, 1999; Moran et al. 1999). In particular, this template spectrum would allow the general possibility of measuring the ongoing star formation rate $(S F R)$ in galaxies from their X-ray spectra, or perhaps - for some galaxies - directly from their X-ray luminosities. The basic notion is that ongoing SFR in a galaxy can be measured based on stellar X-ray sources which are both sufficiently bright for their collective emission to be unambiguously identified, and sufficiently short-lived so that they trace the "instantaneous" SFR. Of the three main types of stellar galactic X-ray sources,

(i) SNRs (which are X-ray bright over timescales $t_{\mathrm{x}} \sim$ $\left.10^{3} \mathrm{yr}\right)$ are the evolutionary outcome of massive $\left(8 \lesssim M / M_{\odot} \lesssim 40\right)$ stars that explode on timescales $(5 \lesssim$ $\tau_{\text {ev }} /$ Myr $\lesssim 50$, see Maeder \& Meynet 1989) short compared with a typical SB duration $\left(\tau_{\mathrm{SB}} \lesssim 100 \mathrm{Myr}\right)$. Hence SNRs do trace the istantaneous $S F R$; however, their relative emission in integrated SFG spectra is quite modest and hard to identify;

(ii) $\operatorname{LMXBs}\left(t_{\mathrm{x}} \sim 10^{7} \mathrm{yr}\right)$ do contribute significantly to the $\mathrm{X}$ ray emission but, due to the long delay between their formation and the onset of their X-ray emission (the donor star has $M \lesssim M_{\odot}$ ), they do not trace the current $S F R$; finally,

(iii) HMXBs $\left(t_{\mathrm{x}} \sim 10^{4} \mathrm{yr}\right)$ provide a suitable combination of short delay between binary formation and onset of X-ray emission (the donor star has $M \gtrsim 8 M_{\odot}$ ) and significant sometimes dominant - relative X-ray emission.

Consequently, the measurement of ongoing $S F R$ in galaxies hinges on our ability to separate out the HMXB contribution from the $2-10 \mathrm{keV}$ luminosity, $L_{2-10}$.

In this paper we extend the work of PR02 by examining ways in which $L_{2-10}$ can be used as an astrophysically motivated $S F R$ estimator for SFGs. The possibility of using $L_{2-10}$ as an integral measure of the $S F R$ has already been suggested (e.g.: Bauer et al. 2002; Grimm et al. 2003; Ranalli et al. 2003; Gilfanov et al. 2004). In this paper we propose that by using the HMXB portion of the hard X-ray luminosity, $L_{2-10}^{\mathrm{HMXB}}$ the $S F R-L_{2-10}^{\mathrm{HMXB}}$ relation is universal and extends from normal galaxies (low $S F R$ ) to very actively starbursting galaxies (very high $S F R$ ). Given the ways in which $L_{2-10}$ can be contaminated, i.e. systematically from LMXBs at low-SFR regimes (PR02), and occasionally but quite frequently from AGNs at high-SFR regimes (Franceschini et al. 2003), our results will emphasize the potential and the limitations of using - over $\sim 5$ decades in $\mathrm{X}$-ray luminosity - the $2-10 \mathrm{keV}$ luminosity as an independent gauge of $S F R$ in SFGs. 
The paper is organized as follows. In Sect. 2 we discuss galactic $S F R$ indicators linked to the presence of short-lived massive $\left(\gtrsim 8 M_{\odot}\right)$ main-sequence stars. Section 3 describes the sample of SFGs, which comprises objects with SBs of various strengths. In Sect. 4 we discuss the effectiveness of the 2-10 keV luminosity of HMXBs as a SFR indicator. Section 5 summarizes our main results. The conclusions are in Sect. 6.

\section{Tracers of ongoing $S F R$}

As mentioned above, the indicators of current SFR are stellar objects or systems whose activity lifetimes are very short compared to characteristic SF timescales. Based on their spectra, luminosities, and numbers, HMXBs are most appropriate $S F R$ gaugers in the $2-10 \mathrm{keV}$ band. In these stellar systems the donor is a massive $\left(M \gtrsim 8 M_{\odot}\right)$ OB star. Now, as a population OB stars are not important contributors to the X-ray output of galaxies (e.g., PR02), but their copious UV emission heats up dusty molecular clouds, with the heat emitted in the FIR. Thus, the $2-10 \mathrm{keV}$ emission of HMXBs and the FIR emission of warm dust clouds are both related to the presence of short-lived OB stars (either isolated or in interacting pairs), with the emission ceasing soon after their death. In principle, then, the clouds' FIR luminosity and the $2-10 \mathrm{keV}$ luminosity of HMXBs can be used independently to measure the instantaneous $S F R$. The crucial step is the separation of the cloud FIR luminosity and the HMXB X-ray luminosity from the corresponding total luminosities: if this can be done properly, the FIR-derived and the X-derived estimates of the SFR should agree.

Massive OB stars found in HMXBs usually end up as supernovae. Shock waves triggered by SN explosions efficiently accelerate electrons to relativistic energies (e.g., Blandford \& Ostriker 1980). Electron energy losses are radiative synchrotron-Compton at energies higher than $\sim 300 \mathrm{MeV}$, with the relative significance of each of these processes depending on the value of the mean magnetic field and the scattering radiation field (either FIR or CMB; see, e.g., Rephaeli et al. 1991). Typical radiative energy loss times are $t_{\text {loss }} \sim 10^{8} \mathrm{yr}$ (e.g., Rephaeli 1979), i.e., comparable to or longer than a typical SB duration. Therefore, galactic radio (synchrotron) emission provides a measure of the $S F R$ averaged over similarly long timescale, and has little to do with the ongoing $S F R$ except in galaxies with constant $S F R$ (see Sect. 3). In the following we shall deal with the $2-10 \mathrm{keV}$ luminosity of HMXBs and the FIR luminosity of dust clouds, both of which are immediate outcomes (and hence tracers) of the current SF activity.

\subsection{Infrared}

In normal disk galaxies, the relationship between the FIR luminosity and the $S F R$ is complex because stars with a variety of ages can contribute to dust heating, and only a fraction of the bolometric luminosity of the young stellar population is absorbed by dust (e.g., Lonsdale Persson \& Helou 1987; Walterbos \& Greenawalt 1996). In SFGs studied here, the physical coupling between the SFR and the IR luminosity is much more direct. Young stars dominate the radiation field that heats the dust, and the dust optical depths are so large that almost all of the bolometric luminosity of the SB is reradiated in the infrared. This makes it possible to derive a reasonable quantitative measure of the $S F R$ from the FIR luminosity.

A popular calibration of the $S F R-L_{\mathrm{FIR}}$ conversion (Kennicutt 1998) is based on the SB synthesis models of Leitherer \& Heckman (1995), which trace the temporal evolution of the bolometric luminosity for a given $S F R$, metal abundance, and IMF. Kennicutt (1998) computed the SFR calibration using their "continuous SF" models, in which the $S F R$ is presumed to remain constant over the lifetime of the burst. The models show that the $L_{\mathrm{bol}} / S F R$ ratio evolves relatively slowly between ages of 10 and $100 \mathrm{Myr}$, the relevant range for most SBs (e.g., Bernlöhr 1993; Engelbracht 1997). Adopting the mean luminosity for the 10-100 Myr continuous bursts, solar abundances, and the Salpeter (1955) stellar initial mass function (IMF) defined as $\mathrm{d} N / \mathrm{d} M \propto M^{-2.35}$ in the mass range $0.1-100 M_{\odot}$, and assuming the bolometric luminosity to be re-radiated by dust, yields ${ }^{2}$ (Kennicutt 1998):

$\operatorname{SFR}\left(\geq 0.1 M_{\odot}\right)=\frac{L_{\mathrm{FIR}}}{5.8 \times 10^{9} L_{\odot}} M_{\odot} \mathrm{yr}^{-1}$.

This lies within the range of published calibrations $(1-3 \times$ $10^{-10} M_{\odot} \mathrm{yr}^{-1}$ ) (e.g. Lehnert \& Heckman 1996; Meurer et al. 1997; Devereux \& Young 1991; see also Inoue et al. 2000 for an analytical derivation, and Silva et al. 1998).

Kennicutt's (1998) relation in Eq. (1) is valid if all the FIR emission is due to warm molecular clouds heated up by newly born massive stars: i.e., Eq. (1) properly applies to starforming regions. If in addition to the warm component there is a "cirrus" component, i.e. emission from (colder) interstellar medium (ISM) heated by the general galactic UV radiation field which is powered also by relatively old stars, then a systematic bias will affect any SFR estimate based on Eq. (1). Any cirrus component should then be filtered out from the observed FIR luminosity, in order to determine the warm emission arising from SF activity. This correction is especially important for objects that are not SB-dominated, such as local normal galaxies and SBGs (where the SB affects only the central disk region), for which the cirrus component is expected to be relatively important. In order to do so we follow David et al. (1992) in using the results of Devereux \& Eales (1989): assuming that the $\mathrm{O}$ stars responsible for FIR emission are (ultimately) linked to particle acceleration (via SN shocks) and (in turn) to nonthermal radio emission, it would be predicted that the cirrus component should be directly proportional to $L_{\mathrm{B}}$ (with the latter a measure of the galactic stellar content), i.e. $L_{\mathrm{FIR}}^{\mathrm{SB}}=L_{\mathrm{FIR}}-x L_{\mathrm{B}}$. Devereux \& Eales (1989) find that if $x=0.14$, then for their sample of 237 galaxies the FIR-radio (i.e., $L_{\mathrm{FIR}}^{\mathrm{SB}}-L_{1.4 \mathrm{GHz}}$ ) correlation is optimized and becomes linear. This implies that, on average, $14 \%$ of the blue luminosity of a normal or starbursting galaxy is reradiated in the FIR band as cirrus emission: for this class of galaxies (see Table 2), the FIR luminosity to be used

2 The FIR flux is defined (Helou et al. 1985) as a combination of the IRAS $60 \mu \mathrm{m}$ and $100 \mu \mathrm{m}$ fluxes according to $f_{\mathrm{FIR}} \equiv 1.26 \times$ $10^{-11}\left(2.58 f_{60}+f_{100}\right) \mathrm{erg} \mathrm{s}^{-1} \mathrm{~cm}^{-1}$, where $f_{60}$ and $f_{100}$ are expressed in Jy. 
in Eq. (1) is then $L_{\mathrm{FIR}}^{\mathrm{SB}}$ (which we here still refer to as $L_{\mathrm{FIR}}$ for simplicity). Anticipating results obtained later in this paper, we can state that allowing for cirrus emission, while theoretically required and practically favorable (doing so only strengthens our results), amounts only to a minor correction.

\subsection{X-rays}

Being able to ascertain whether the $2-10 \mathrm{keV}$ band is dominated by HMXBs or LMXBs may yield substantial insight on the SF history of a galaxy. In a SB environment the formation of low-mass stars could be suppressed if SN blast waves of more rapidly forming massive stars disrupt the slowly forming less massive stars before these complete (or even reach) their Hayashi tracks. The ensuing stellar IMF would be top-heavy (see, e.g., Doane \& Mathews 1993; Rieke et al. 1993 for M 82), in which case no LMXBs would form, and the resulting binary population of the SB consists only of HMXBs. But even if the IMF were not top-heavy, one isolated SB episode would not trigger an increase of the LMXB population. In fact, the time required for the $\lesssim 1 M_{\odot}$ optical companion in a LMXB system to evolve out of the main sequence and come into Roche-lobe contact (and hence start the X-ray bright phase) substantially exceeds a typical SB lifetime $\left(\tau_{\mathrm{SB}} \lesssim 10^{8} \mathrm{yr}\right)$. So, whatever is the mass range of the relevant stellar IMF, during one isolated SB episode there is time only for HMXBs (i.e., accretionpowered X-ray pulsars) to form (in larger numbers for a topheavy IMF). The resulting 2-10 keV synthetic spectrum would then be dominated by HMXBs, and hence the SB spectral slope would be characterized by a flat photon index $\Gamma \simeq 1.2$ (see Fig. 1-right $)^{3}$.

In the case of recurrent SF bursts, LMXBs could be important contributors in the $2-10 \mathrm{keV}$ band. This may happen in, e.g., galaxies that are members of pairs with highly eccentric orbits, or that are found in crowded environments (e.g., compact groups: see Hickson et al. 1989; see also Della Ceca et al. 1997). In both cases, tidal interactions would be recurrent, so bright LMXB could be present as leftovers from earlier interactions. If one such galaxy is observed in a post-SB (or quiescent) phase, the $2-10 \mathrm{keV}$ emission would practically come only from LMXBs.

Therefore, determining the type of X-ray binaries whose emission dominates the $2-10 \mathrm{keV}$ luminosity of SFGs could provide a clue both to the understanding of SF history of those galaxies (e.g., Holt et al. 2003), and to using $L_{2-10}$ as an indicator of the ongoing SFR. As an example of the latter, let us consider three representative cases of SFGs.

(i) In a galaxy where the SF activity has been constant over several $10^{8} \mathrm{yr}$, the relative frequencies of occurrence of

\footnotetext{
${ }^{3}$ Spectral components that are flat in the $2-10 \mathrm{keV}$ band can come from AGNs. Both a direct nuclear emission (with $\Gamma \sim 2$ ) that is heavily absorbed, or an emission that is reflected by a warm medium, are observed as relatively flat $(\Gamma \sim 1.2)$ spectra (e.g., NCC 6240, NGC 4945, Arp 299: Vignati et al. 1999; Guainazzi et al. 2000; Della Ceca et al. 2002). In general there will be no degeneracy between the AGN and HMXB interpretations of the flat spectral components, as the inferred luminosity should enable a clear identification.
}

stellar X-ray sources (SNRs, HMXBs, LMXBs) are expected to agree with those predicted for the Galaxy (which has a $S F R$ of $\sim 2 M_{\odot} \mathrm{yr}^{-1}$ ) by the Iben et al. (1995a,b) models, upon which the PR02 template X-ray spectrum is based. By comparing the deduced HMXB content of a given galaxy (from X-ray spectral decomposition) with that of the Galaxy, its current $S F R$ can be estimated;

(ii) for SB-dominated galaxies the above considerations suggest that $L_{2-10}$ is an indicator of $S F R$;

(iii) in the intermediate case of a galaxy undergoing a minor SB (which is usually located in the central region), $L_{2-10}$ is a superposition of the emissions from the (quiescent) bulge/disk and the ongoing SB. Only after these two components are separated out, can the "quiescent" and the "bursting" SFRs be estimated (see Fig. 2).

\section{The samples}

To test the idea that the $S F R-L_{\mathrm{x}}$ relation largely reflects the ability to use HMXBs to trace the current $S F R$, we have selected three representative samples of SFGs.

The sample of ULIRGs (Table 1) - sources with bolometric luminosity $>10^{12} L_{\odot}$ emitted mostly in the IR $(8-1000 \mu \mathrm{m})$ band - is essentially the list of IRAS-selected galaxies that represent some of the most luminous galaxies in the local Universe observed with ISO by Genzel et al. (1998), whose FIR selection makes it unbiased with respect to absorption. It is fluxlimited and complete down to $S_{60 \mu \mathrm{m}} \geq 5.4 \mathrm{Jy}$, and highquality IR/optical spectroscopic data are available for all the sources (Lutz et al. 1999; Veilleux et al. 1999). X-ray data in both the soft and hard bands are from ASCA, BeppoSAX, and $X M M-N e w t o n$. According to the most extensive spectral survey of ULIRGs to date (Franceschini et al. 2003), the ULIRG spectra are qualitatively similar to SBG spectra, plus possibly (in $40 \%$ cases) a heavily absorbed PL component that shows up at $>5 \mathrm{keV}$ (rest-frame). A similar incidence rate of AGNs in ULIRGs is deduced using mid-IR diagnostics (Genzel et al. 1998).

The sample of SBGs (Table 2), compiled from Genzel et al. (1998) and Ranalli et al. (2003), consists of local FIR-bright objects known to contain central starbursts of various strengths for which X-ray spectral information is available (from Einstein, ROSAT, ASCA, BeppoSAX, and RossiXTE). It should be stressed that this sample comprises a fair number of "normal" galaxies, i.e. galaxies whose $S F R$ is comparable to that of the Milky Way. These SFGs are useful for checking the $S F R-L_{\mathrm{x}}$ relation at the low-SFR end of the galaxy distribution. A more appropriate name for this sample should then be "local SFGs" (of which SBGs are, technically speaking, a subset); but for lack of a definite, clear-cut distinction between "normal" and "starburst" galaxies, in this paper we'll stick to the denomination "SBG sample" in order to emphasize that our main interest for these galaxies lies in their SF activity. Judging by currently available X-ray measurements, most of these galaxies share a remarkable spectral homogeneity with their spectra usually described (most simply) as a soft thermal component 
Table 1. Data I: the sample of ultra-luminous IR galaxies (ULIRGs $)^{a}$.

\begin{tabular}{|c|c|c|c|c|c|c|c|}
\hline Object & $\begin{array}{l}D^{b} \\
{[\mathrm{Mpc}]}\end{array}$ & $\begin{array}{l}f_{0.5-2} \\
{\left[10^{-14} \mathrm{erg} \mathrm{s}^{-1}\right]}\end{array}$ & $\begin{array}{l}f_{2-10} \\
{\left[10^{-14} \mathrm{erg} \mathrm{s}^{-1}\right]}\end{array}$ & Instr. & $\begin{array}{l}f_{60} \\
{[\mathrm{Jy}]}\end{array}$ & $\begin{array}{l}f_{100} \\
{[\mathrm{Jy}]}\end{array}$ & Notes $^{c}$ \\
\hline IRAS 05189-2524 & $170^{[7]}$ & $\ldots$ & $360.0^{[7]}$ & SAX & $13.25^{[8]}$ & $11.84^{[8]}$ & $\mathrm{AGN}^{[7]}$ \\
\hline IRAS $12112+0305$ & $291^{[3]}$ & $1.53^{[2]}$ & $1.62^{[2]}$ & XMM & $8.18^{[3]}$ & $9.46^{[3]}$ & SB \\
\hline Mkn 231 & $169^{[3]}$ & $29.06^{[2,11]}$ & $84.54^{[2,11]}$ & XMM & $32.0^{[3]}$ & $30.3^{[3]}$ & $\mathrm{SB}+\mathrm{AGN}^{[2]}$ \\
\hline Mkn 273 & $151^{[3]}$ & $17.00^{[4]}$ & $70.0^{[4]}$ & ASCA & $21.7^{[3]}$ & $21.4^{[3]}$ & $\mathrm{SB}+\mathrm{AGN}^{[4]}$ \\
\hline IRAS $14348-1447$ & $330^{[3]}$ & $3.08^{[2]}$ & $1.91^{[2]}$ & XMM & $6.82^{[3]}$ & $7.31^{[3]}$ & SB \\
\hline IRAS $15250-3609$ & $213^{[3]}$ & $2.31^{[2]}$ & $2.31^{[2]}$ & XMM & $7.10^{[3]}$ & $5.93^{[3]}$ & SB \\
\hline Arp 220 & $73^{[3]}$ & $8.0^{[6]}$ & $18.0^{[6]}$ & SAX & $104.0^{[3]}$ & $112.0^{[3]}$ & SB \\
\hline NGC 6240 & $97^{[3]}$ & $64.0^{[5]}$ & $190.0^{[5,9]}$ & ASCA, SAX & $22.94^{[3]}$ & $26.49^{[3]}$ & $\mathrm{AGN}^{[9]}$ \\
\hline IRAS $17208-0014$ & $170^{[3]}$ & $7.56^{[2]}$ & $4.54^{[2]}$ & XMM & $9.53^{[3]}$ & $11.05^{[3]}$ & SB \\
\hline IRAS $19254-7245$ & $246^{[3]}$ & $10.91^{[2,10]}$ & $17.58^{[2,10]}$ & XMM & $5.5^{[3]}$ & $5.8^{[3]}$ & $\begin{array}{l}\mathrm{SB}+\mathrm{AGN}^{[2]} \\
\text { (The Superantennae) }\end{array}$ \\
\hline IRAS 20100-4156 & $517^{[3]}$ & $1.68^{[2]}$ & $2.24^{[2]}$ & XMM & $5.2^{[3]}$ & $5.2^{[3]}$ & SB \\
\hline IRAS $20551-4250$ & $171^{[3]}$ & $52.93^{[2]}$ & $99.56^{[2]}$ & XMM & $12.8^{[3]}$ & $10.0^{[3]}$ & $\mathrm{SB}+\mathrm{AGN}^{[2]}$ \\
\hline IRAS $22491-1808$ & $309^{[3]}$ & $0.61^{[2]}$ & $0.65^{[2]}$ & XMM & $5.54^{[3]}$ & $4.64^{[3]}$ & SB \\
\hline IRAS $23060+0505$ & $692^{[3]}$ & $270.00^{[1]}$ & $250.0^{[1]}$ & ASCA & $1.2^{[3]}$ & $0.8^{[3]}$ & $\mathrm{AGN}^{[1]}$ \\
\hline IRAS $23128-5919$ & $178^{[3]}$ & $12.04^{[2]}$ & $21.66^{[2]}$ & XMM & $10.8^{[3]}$ & $11.0^{[3]}$ & $\mathrm{SB}+\mathrm{AGN}^{[2]}$ \\
\hline
\end{tabular}

${ }^{a}$ References: [1] Brandt et al. (1997); [2] Franceschini et al. (2003); [3] Genzel et al. (1998); [4] Iwasawa (1999); [5] Iwasawa \& Comastri (1998); [6] Iwasawa et al. (2001); [7] Severgnini et al. (2001); [8] Sanders et al. (2003); [9] Vignati et al. (1999); [10] Braito et al. (2003); [11] Braito et al. (2004).

${ }^{b}$ Distances are taken from Genzel et al. (1998), or are computed consistently assuming $H_{0}=75 \mathrm{~km} \mathrm{~s}^{-1} \mathrm{Mpc}^{-1}, q_{0}=0.5$ otherwise.

${ }^{c}$ Component(s) dominating the $2-10 \mathrm{keV}$ emission; other name(s).

at $<1 \mathrm{keV}$, plus a hard (sometimes cutoff) PL at $>2 \mathrm{keV}$ (e.g., Dahlem et al. 1998).

The sample of Hubble Deep Field North (HDFN) galaxies (Table 3) was selected by Ranalli et al. (2003) from the $1 \mathrm{Ms}$ Chandra (Brandt et al. 2001) and radio (1.4 GHz: Richards 2000 and Garrett et al. 2000) catalogues of the HDFN, which reaches limiting fluxes low enough for SFGs at $z \sim 1.3$ to be detected. As emphasized by Ranalli et al. (see also Grimm et al. 2003), these galaxies can be used to investigate the high- $z$ behaviour of the $S F R-\mathrm{X}$-ray luminosity relation, which is being explored primarily in the local universe $(z \lesssim 0.1)$. Lacking FIR data, we estimate the $S F R$ from the radio flux density at $1.4 \mathrm{GHz}$ using the relation $\operatorname{SFR}\left(\geq 5 M_{\odot}\right)=L_{1.4 \mathrm{GHz}} /(4 \times$ $\left.10^{28} \mathrm{erg} \mathrm{s}^{-1} \mathrm{~Hz}^{-1}\right) M_{\odot} \mathrm{yr}^{-1}$ (Condon 1992), with the resulting $S F R$ are reported in Table 3. It should be emphasized that the Condon formula yields the formation rate of stars with $5 \leq M / M_{\odot} \leq 100$. To compare these $S F R$ estimates with those we use for the SBG and ULIRG samples, we must extrapolate the radio $S F R$ down to $0.1 M_{\odot}$ using the Salpeter (1955) stellar IMF upon which the Kennicutt (1998) formula is based. Doing so the radio and FIR estimates will be consistent ${ }^{4}$. We then obtain $\operatorname{SFR}\left(\geq 0.1 M_{\odot}\right)=5.5 \operatorname{SFR}\left(\geq 5 M_{\odot}\right)$. We will use these new values, $S F R\left(\geq 0.1 M_{\odot}\right)$, as the reference $S F R$ values for our HDFN galaxies. It should be pointed out that, owing to the long lifetime of the radio-emitting relativistic electrons, $\sim 10^{9} \mathrm{yr}$, radio emission may not be able to trace shortertimescale SBs (see Sect. 2). However, it is realistic to assume that HDFN galaxies, being in an earlier evolutionary phase than

\footnotetext{
${ }^{4}$ In the derivation of the $S F R-L_{1.4 \mathrm{GHz}}$ conversion, Condon (1992) used a Salpeter-like IMF with index 2.50 , instead of 2.35 as adopted by Kennicutt (1998). For our purposes here this difference is negligible.
}

local galaxies, are dominated by strong continuous SF activity (e.g.: Madau et al. 1996; Thompson et al. 2001) and hence their $S F R$ can be effectively measured by the non-thermal radio emission.

\section{HMXBs as gauges of the $S F R$}

We now demonstrate that the HMXB portion of the $2-10 \mathrm{keV}$ luminosity, $L_{2-10}^{\mathrm{HMXB}}$, can be used as a gauge of the $S F R$. To this end we compare the $S F R$ computed from the FIR according to the Kennicutt (1988) formula (see Eq. (1)) with the SFR estimated from the integrated luminosity of the bright-HMXB population. We assume a mean luminosity of $5 \times 10^{37} \mathrm{erg} \mathrm{s}^{-1}$ for bright HMXBs. For each sample galaxy, we estimate the number of HMXBs from the $L_{2-10}^{\mathrm{HMXB}}$. By comparison with the Galaxy, which hosts $\sim 50$ bright HMXBs (Iben et al. 1995a, and references therein), and has $S R F \sim 2 M_{\odot} \mathrm{yr}^{-1}$ (e.g., Matteucci 2002), we estimate the current $S F R$ of the galaxy in question. This simple procedure is applied to both the ULIRG and SBG samples.

- ULIRGs. If $L_{2-10}$ is used, the X-ray estimates are in overall agreement with the FIR estimates for the AGN-free ULIRGs (Fig. 4: filled dots), while they are clearly in excess for objects whose $2-10 \mathrm{keV}$ emission is contaminated, or dominated, by a central $\mathrm{AGN}^{5}$ (Fig. 4: empty dots and crosses, respectively). The overall agreement between FIR-derived and X-derived estimates of the SFR suggests that the $2-10 \mathrm{keV}$ luminosity of

\footnotetext{
5 With both $S F R$ estimates linear in the respective luminosities, an equivalent statement is that, for a given $L_{\mathrm{FIR}}$, AGN-contaminated ULIRGs are X-ray overluminous compared with SB-dominated ones; see also Ptak et al. (2003).
} 
Table 2. Data II: the sample of local normal and starburst galaxies (SBG sample) $)^{a}$.

\begin{tabular}{|c|c|c|c|c|c|c|c|c|}
\hline Object $^{b}$ & $\begin{array}{l}D^{c} \\
{[\mathrm{Mpc}]}\end{array}$ & $\begin{array}{l}f_{0.5-2}^{d} \\
{\left[10^{-12} \mathrm{erg} \mathrm{s}^{-1}\right]}\end{array}$ & $\begin{array}{l}f_{2-10} \\
{\left[10^{-12} \mathrm{erg} \mathrm{s}^{-1}\right]}\end{array}$ & Instr. & $B_{\mathrm{T}}^{0 e}$ & $\begin{array}{l}f_{60} \\
{[\mathrm{Jy}]}\end{array}$ & $\begin{array}{l}f_{100} \\
{[\mathrm{Jy}]}\end{array}$ & Notes $^{f}$ \\
\hline NGC $0055^{\mathrm{R}}$ & $1.3^{[26]}$ & $1.8^{[2,20]}$ & $0.68^{[2,20]}$ & ROSAT, ASCA & 7.63 & $77.00^{[8]}$ & $174.09^{[8]}$ & \\
\hline NGC $0253^{\mathrm{GR}}$ & $3.0^{[26]}$ & $2.5^{[1,20]}$ & $5.0^{[1,20]}$ & SAX & 7.09 & $967.81^{[8]}$ & $1288.15^{[8]}$ & \\
\hline NGC 0628 & $9.7^{[26]}$ & $\ldots$. & $0.249^{[15]}$ & ASCA & 9.76 & $21.54^{[8]}$ & $54.45^{[8]}$ & \\
\hline NGC $0891^{R}$ & $9.6^{[26]}$ & $0.83^{[20]}$ & $1.9^{[20]}$ & ASCA & 9.37 & $66.46^{[8]}$ & $172.23^{[8]}$ & \\
\hline NGC $1569^{R}$ & $1.6^{[26]}$ & $0.54^{[3]}$ & $0.22^{[3]}$ & ASCA & 9.42 & $54.36^{[8]}$ & $55.29^{[8]}$ & \\
\hline NGC $1808^{R}$ & $10.8^{[26]}$ & $0.65^{[20]}$ & $0.76^{[20]}$ & ASCA & 10.43 & $105.55^{[8]}$ & $141.76^{[8]}$ & \\
\hline NGC $2146^{\mathrm{R}}$ & $17.2^{[26]}$ & $0.82^{[5]}$ & $1.11^{[5]}$ & ASCA & 10.58 & $146.69^{[8]}$ & $194.05^{[8]}$ & \\
\hline NGC $2276^{R}$ & $36.8^{[26]}$ & $0.21^{[20]}$ & $0.44^{[20]}$ & ASCA & 11.75 & $14.29^{[8]}$ & $28.97^{[8]}$ & \\
\hline NGC $2403^{R}$ & $4.2^{[26]}$ & $1.6^{[20]}$ & $0.93^{[20]}$ & ASCA & 8.43 & $41.47^{[8]}$ & $99.13^{[8]}$ & \\
\hline NGC 2782 & $37.3^{[26]}$ & $1.3^{[25]}$ & $\ldots$. & ROSAT & 12.01 & $9.17^{[8]}$ & $13.76^{[8]}$ & \\
\hline NGC $2903^{R}$ & $6.3^{[26]}$ & $0.79^{[20]}$ & $0.686^{[15]}$ & ASCA & 9.11 & $60.54^{[8]}$ & $130.43^{[8]}$ & \\
\hline NGC $3034^{\mathrm{GR}}$ & $5.2^{[26]}$ & $5.8^{[21]}$ & $15.5^{[21]}$ & RXTE & 5.58 & $1480.42^{[8]}$ & $1373.69^{[8]}$ & $=\mathrm{M} 82$ \\
\hline NGC 3079 & $20.4^{[26]}$ & $4.58^{[2]+}$ & $0.78^{[2]}$ & ROSAT, ASCA & 10.41 & $50.67^{[8]}$ & $104.69^{[8]}$ & \\
\hline NGC $3256^{\mathrm{GR}}$ & $37.4^{[26]}$ & $0.69^{[16]}$ & $0.586^{[16]}$ & ASCA & 8.34 & $102.63^{[8]}$ & $114.31^{[8]}$ & \\
\hline NGC 3310 & $18.7^{[26]}$ & $0.74^{[20]}$ & $0.21^{[27]}$ & ASCA & 10.95 & $34.56^{[8]}$ & $48.19^{[8]}$ & \\
\hline NGC $3367^{R}$ & $43.6^{[26]}$ & $0.18^{[20]}$ & $0.16^{[20]}$ & ASCA & 11.92 & $6.44^{[8]}$ & $13.48^{[8]}$ & \\
\hline NGC $3556^{\mathrm{R}}$ & $14.1^{[26]}$ & $0.44^{[20]}$ & $0.60^{[20]}$ & ASCA & 9.83 & $32.55^{[8]}$ & $76.90^{[8]}$ & $=\mathrm{M} 108$ \\
\hline NGC 3628 & $7.7^{[26]}$ & $4.16^{[2]+}$ & $0.98^{[2]}$ & ROSAT, ASCA & 9.31 & $54.80^{[8]}$ & $105.76^{[8]}$ & \\
\hline $\operatorname{Arp} 299^{\mathrm{GR}}$ & $41.6^{[17]}$ & $0.57^{[20]}$ & $1.08^{[20,23]}$ & ASCA & 11.85 & $113.05^{[8]}$ & $111.42^{[8]}$ & $\begin{array}{l}=\mathrm{NGC} 3690+\mathrm{IC} 694 \\
\text { AGN at } \gtrsim 10 \mathrm{keV}^{[32]}\end{array}$ \\
\hline NGC $4038 / 39^{G R}$ & $25.4^{[26]}$ & $0.72^{[20,23]}$ & $0.53^{[20,23]}$ & ASCA & 10.62 & $45.16^{[8]}$ & $87.09^{[8]}$ & $=$ The Antennae \\
\hline NGC $4449^{\mathrm{R}}$ & $3.0^{[26]}$ & $0.826^{[4]}$ & $0.482^{[4]}$ & ASCA & 9.94 & $36.0^{[11]}$ & $73.0^{[11]}$ & \\
\hline $\mathrm{NGC} 4631^{\mathrm{R}}$ & $6.9^{[26]}$ & $40.0^{[2]+}$ & $1.15^{[2]}$ & ROSAT, ASCA & 8.61 & $85.40^{[8]}$ & $160.08^{[8]}$ & \\
\hline NGC $4654^{R}$ & $16.8^{[26]}$ & $0.06^{[20]}$ & $0.09^{[20]}$ & ASCA & 10.75 & $13.39^{[8]}$ & $37.77^{[8]}$ & \\
\hline NGC 4666 & $14.1^{[26]}$ & $0.16^{[19]}$ & $0.29^{[19]}$ & SAX & 10.68 & $37.11^{[8]}$ & $85.95^{[8]}$ & \\
\hline NGC $4945^{\mathrm{G}}$ & $5.2^{[26]}$ & $1.3^{[10]}$ & $5.4^{[10]}$ & SAX & 7.43 & $625.46^{[8]}$ & $1329.70^{[8]}$ & $\begin{array}{l}\text { AGN } \\
\text { at } \gtrsim 10 \mathrm{keV}^{[10,31,32,22,24]}\end{array}$ \\
\hline NGC $5236^{\mathrm{G}}$ & $4.7^{[26]}$ & $3.5^{[18]}$ & $4.7^{[18]}$ & ASCA & 7.98 & $265.84^{[8]}$ & $524.09^{[8]}$ & $=\mathrm{M} 83$ \\
\hline NGC $5253^{\mathrm{G}}$ & $3.2^{[26]}$ & $0.32^{[14]}$ & $\ldots$ & ROSAT & 10.47 & $29.84^{[8]}$ & $30.08^{[8]}$ & \\
\hline NGC $5457^{\mathrm{R}}$ & $5.4^{[26]}$ & $0.54^{[20]}$ & $0.68^{[20]}$ & ASCA & 8.21 & $88.04^{[8]}$ & $252.84^{[8]}$ & $=\mathrm{M} 101$ \\
\hline NGC $6946^{R}$ & $5.5^{[26]}$ & $3.0^{[20]}$ & $1.2^{[20]}$ & ASCA & 7.78 & $129.78^{[8]}$ & $290.69^{[8]}$ & \\
\hline NGC $7469^{\mathrm{G}}$ & $65.2^{[17]}$ & $\ldots$. & $29.8^{[9]}$ & ASCA & 12.64 & $27.33^{[8]}$ & $35.16^{[8]}$ & $\mathrm{AGN}^{[28,29,30]}$ \\
\hline $\mathrm{NGC} 7552^{\mathrm{G}}$ & $19.5^{[26]}$ & $1.0^{[13]}$ & $0.36^{[13]}$ & Einstein & 11.13 & $77.37^{[8]}$ & $102.92^{[8]}$ & \\
\hline NGC 7679 & $71.0^{[6]}$ & $3.3^{[6]}$ & $6.0^{[6]}$ & SAX & 12.89 & $7.40^{[8]}$ & $10.71^{[8]}$ & $\mathrm{AGN}^{[6]}$ \\
\hline IC $342^{\mathrm{R}}$ & $3.9^{[26]}$ & $1.8^{[20]}$ & $11.0^{[20]}$ & ASCA & 6.04 & $180.80^{[8]}$ & $391.66^{[8]}$ & \\
\hline
\end{tabular}

${ }^{a}$ References: [1] Cappi et al. (1999); [2] Dahlem et al. (1998); [3] Della Ceca et al. (1996); [4] Della Ceca et al. (1997); [5] Della Ceca et al. (1999); [6] Della Ceca et al. (2001); [7] de Naray et al. (2000); [8] Sanders et al. (2003); [9] Guainazzi et al. (1994); [10] Guainazzi et al. (2000); [11] Hunter et al. (1986); [12] Iwasawa et al. (1993); [13] Maccacaro \& Perola (1981); [14] Martin \& Kennicutt (1995); [15] Mizuno et al. (1998); [16] Moran et al. (1999); [17] NED; [18] Okada et al. (1990); [19] Persic et al. (2003); [20] Ranalli et al. (2003); [21] Rephaeli \& Gruber (2002); [22] Done et al. (2003); [23] Sansom et al. (1996); [24] Schurch et al. (2002); [25] Schulz et al. (1998); [26] Tully (1988); [27] Zezas et al. (1998); [28] Perez-Olea \& Colina (1996); [29] Nandra et al. (2000); [30] Blustin et al. (2003); [31] Madejski et al. (2000); [32] Della Ceca et al. (2002).

${ }^{b}$ The superscripts G, R indicate whether an object is included in the Genzel et al. (1998) or Ranalli et al. (2003) samples, respectively.

${ }^{c}$ Distances are taken from Tully (1988) if $c z \leq 3000 \mathrm{~km} \mathrm{~s}^{-1}$, or are computed consistently assuming $H_{0}=75 \mathrm{~km} \mathrm{~s}^{-1} \mathrm{Mpc}^{-1}$ otherwise.

${ }^{d}$ Fluxes marked with a cross refer to the $0.1-2.0 \mathrm{keV}$ band.

${ }^{e}$ Blue apparent magnitudes, corrected to face-on and for Galactic absorption, from RC3 (de Vaucouleurs et al. 1991).

${ }^{f}$ Other name(s); spectrally dominating component(s) at energies $\gtrsim 10 \mathrm{keV}$.

AGN-free ULIRGs is indeed (mainly) due to HMXB: this conclusion is in accord with their flat observed $2-10 \mathrm{keV}$ continua, which are consistent with the $\Gamma \sim 1.2$ spectra observed in Galactic HMXBs.

For the objects in which the SB and AGN contributions to the 2-10 keV flux are comparable (see Table 1), we employed the following crude procedure in order to estimate the HMXB contribution, a procedure that is admittedly quite arbitrary given the uncertain observational situation, but that nonetheless has some theoretical justification. For the $4 \mathrm{ob}-$ jects taken from Franceschini et al. (2003), we have added a $\Gamma \sim 1.2$ PL component to the published spectral models ${ }^{6}$, repeating the $\chi^{2}$ minimization. Results of the fitting, while not

${ }^{6}$ IRAS 19254-7245: Braito et al. (2003); IRAS 20551-4250, IRAS 23128-5919: Franceschini et al. (2003); and Mkn 231: Braito et al. (2004). 
Table 3. Data III: the sample of Hubble Deep Field North galaxies (HDFN sample) ${ }^{a}$.

\begin{tabular}{llllllll}
\hline \hline Source & $z$ & $\begin{array}{l}F_{1.4 \mathrm{GHz}} \\
{[\mu \mathrm{Jy}]}\end{array}$ & $\begin{array}{l}L_{1.4 \mathrm{GHz}} \\
{\left[\mathrm{erg} \mathrm{s}^{-1} \mathrm{~Hz}^{-1}\right]}\end{array}$ & $\begin{array}{l}S F R^{b} \\
{\left[M_{\odot} \mathrm{yr}^{-1}\right]}\end{array}$ & $\begin{array}{l}f_{2-10} \\
{\left[\mathrm{erg} \mathrm{s}^{-1} \mathrm{~cm}^{-2}\right]}\end{array}$ & $\begin{array}{l}L_{2-10} \\
{\left[\mathrm{erg} \mathrm{s}^{-1}\right]}\end{array}$ & Instr. \\
\hline 134 & 0.456 & 210 & $1.03 \times 10^{30}$ & 25.8 & $2.8 \times 10^{-16}$ & $1.38 \times 10^{41}$ & Chandra \\
136 & 1.219 & 180 & $8.68 \times 10^{31}$ & 217.1 & $1.9 \times 10^{-16}$ & $9.17 \times 10^{41}$ & Chandra \\
148 & 0.078 & 96 & $1.16 \times 10^{28}$ & 0.3 & $<4.1 \times 10^{-17}$ & $<4.95 \times 10^{38}$ & Chandra \\
188 & 0.410 & 83 & $3.23 \times 10^{29}$ & 8.1 & $5.8 \times 10^{-17}$ & $2.26 \times 10^{40}$ & Chandra \\
194 & 1.275 & 60 & $3.24 \times 10^{30}$ & 80.9 & $2.0 \times 10^{-16}$ & $1.08 \times 10^{42}$ & Chandra \\
246 & 0.423 & 36 & $1.50 \times 10^{29}$ & 3.8 & $7.5 \times 10^{-17}$ & $3.13 \times 10^{40}$ & Chandra \\
278 & 0.232 & 160 & $1.84 \times 10^{29}$ & 4.6 & $1.6 \times 10^{-16}$ & $1.84 \times 10^{40}$ & Chandra \\
\hline
\end{tabular}

${ }^{a}$ All data are taken, or derived, from Ranalli et al. (2003). Distant dependent quantities have been computed assuming $H_{0}=75 \mathrm{~km} \mathrm{~s}{ }^{-1}$ and $q_{0}=0.5$.

${ }^{b}$ The $S F R$ has been computed using Condon's (1992) formula $S F R\left(>5 M_{\odot}\right)=L_{1.4 \mathrm{GHz}} /\left(4 \times 10^{28} \mathrm{erg} \mathrm{s}^{-1} \mathrm{~Hz}^{-1}\right) M_{\odot} \mathrm{yr}^{-1}$, which yields the $S F R$ for stars in the mass range $5 \leq M / M_{\odot} \leq 100$ (assuming a Salpeter-like stellar IMF with $\mathrm{d} N / \mathrm{d} M \propto M^{-2.5}$, and a non-thermal radio spectral index $\alpha=0.8)$.

compelling because the quality of the data allows only simple model testing, do indicate a roughly $\sim 10 \%$ HMXBs contribution to the $2-10 \mathrm{keV}$ flux. Specifically, the additional PL fluxes are $f_{2-10}=2.06,1.89,1.44$, and $9.25 \times 10^{-14} \mathrm{erg} \mathrm{cm}^{-2} \mathrm{~s}^{-1}$, respectively (in the order listed in footnote 6), which in turn correspond to luminosities of $L_{2-10}=1.49 \times 10^{41}, 6.59 \times 10^{40}$, $5.44 \times 10^{40}$, and $3.15 \times 10^{41} \mathrm{erg} \mathrm{s}^{-1}$ (see Fig. 3). For Mkn 273 we have followed the argument of Xia et al. (2002) who, in their analysis of the Chandra spectrum, have identified a moderately absorbed PL component which they suggest must be at least partially associated with HMXBs. (This PL is in addition to a steeper and more absorbed PL that Xia et al. associate with the the direct light from the central AGN.) Therefore, lacking more detailed information, we assume the HMXB contribution in Mkn 273 to be half of the flux in the less-absorbed PL, i.e. $f_{2-10}=4.6 \times 10^{-14} \mathrm{erg} \mathrm{cm}^{-2} \mathrm{~s}^{-1}$ corresponding to $L_{2-10}=1.27 \times 10^{41} \mathrm{erg} \mathrm{s}^{-1}$. We emphasize that for the five AGN-contaminated ULIRGs of our sample the estimated HMXB contributions are very preliminary, given the substantial uncertainties. However, it is remarkable that the "corrected" luminosities cluster so closely with the total 2-10 keV luminosities of the AGN-free objects (Fig. 5-right: empty dots).

- SBGs. In comparing SBGs and ULIRGs, we should bear in mind a main difference between the two classes that is particularly relevant to our discussion here. In ULIRGs the star forming activity is generally very intense and involves most of the stellar disks; in local SBGs (such as those included in Table 2), SF is considerably less intense and occurs mainly in the very central disk region. Thus, for non spatially resolved measurements, such as the ASCA and BeppoSAX data used in Figs. 4 and 5 , the $2-10 \mathrm{keV}$ emission is expected to come mostly from HMXBs in ULIRGs, and from a mix of HMXBs (in the central starbursting region) and LMXBs (in the underlying quiescent disk) in normal and staburst galaxies. Therefore, if this effect is not corrected for, our X-ray-based estimate of the $S F R$, while fairly adequate in principle (albeit crude) in the case of ULIRGs, is systematically overestimated in the SBG sample by a factor $1 / f$, with $f$ the HMXB-to-total luminosity ratio. Thus, we need to estimate $f$ for the SBG sample listed in Table 2.

To this end we fit the template X-ray model of PR02 (see Fig. 1-left), keeping the profiles of the spectral components frozen, and letting only the amplitudes free to vary, to available data for such galaxies. In principle this procedure should be applied to each and every galaxy in the sample. In practice, however, for most of the objects the available data are such that it is difficult even to determine whether the harder spectral component is PL or thermal (e.g., Dahlem et al. 1998, 2000), let alone a more complicated multi-component (thermal and PL) model including also emission from HMXBs, SNRs, and LMXBs. The very nearby M 82, which has been observed with $A S C A$ in the $0.4-10 \mathrm{keV}$ range (Ptak et al. 1997) and BeppoSAX in the 0.1-60 keV range (Cappi et al. 1999), is currently the best example of a relatively well-observed SBG. In Fig. 2 we show the result of fitting the PR02 template to the combined $A S C A+B$ eppoSAX data (see the Appendix). The results of these fits yield $f \sim 0.2$.

In order to check to what extent this value of $f$ may be taken as representative of SBGs in general, we have performed additional fits for a subset of our SBG objects (including M 83, NGC 253, NGC 2146, NGC 2903, NGC 3310, NGC 3256) being aware, however, that the archival ASCA spectra available for this analysis are inferior to that of M 82, so any result from such a comparison is necessarily preliminary and merely suggestive. (For the other objects of the SBG sample for which ASCA data exist, we felt that the quality of the data was not sufficient for even a rough estimation of $f$.) In addition to one or more sub$\mathrm{keV}$ thermal plasma components, the fitting models we used did include a HMXB component plus either a plain/cutoff PL (NGC 2146, NGC 2903, NGC 3256, NGC 3310), or the explicit SNR+LMXB components (M 83, NGC 253). Within the limits imposed by the relatively poor statistics of these data, the results are consistent with $f \sim 0.2$ for the objects with highest quality data (M 83, NGC 253, NGC 2903), while suggesting a slightly higher value of $f$ in the other cases (NGC 2146, NGC 3256). The exception seems to be NGC 3310, whose hard 


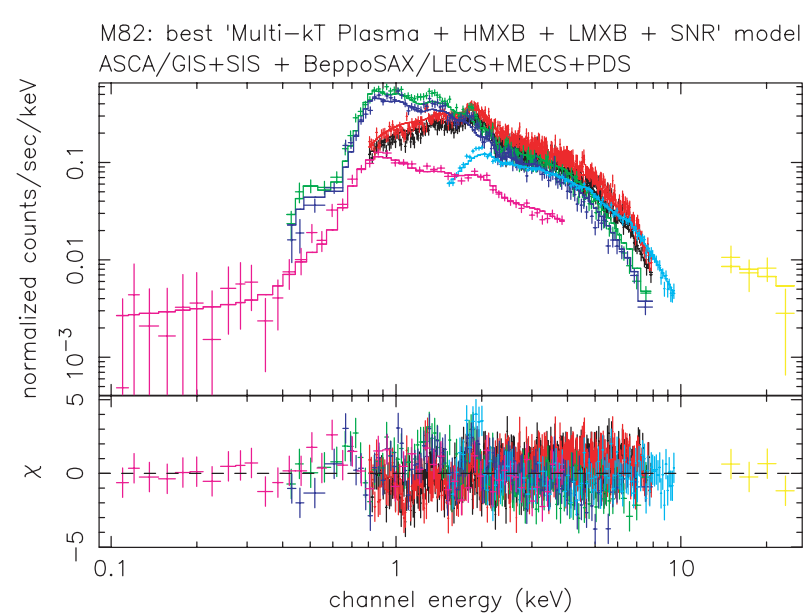

M82: 'Multi-kT Plasma + LMXB + HMXB + SNR' model ASCA + BeppoSAX data

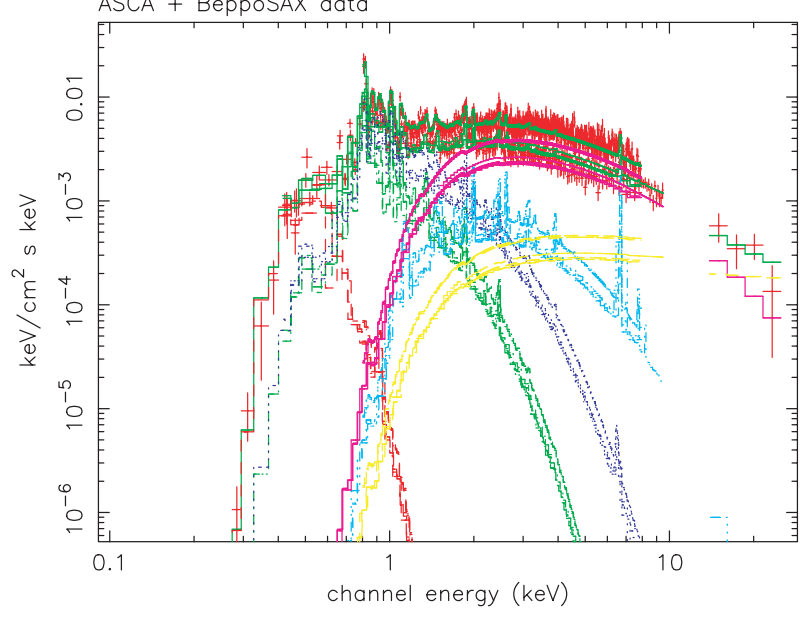

Fig. 2. The "standard" model shown in Fig. 1-left is fitted to the combined ASCA and BeppoSAX data of M 82 (ASCA: black, red, green, and dark-blue points; BeppoSAX: purple, cyan, and yellow points). The profiles of the spectral components are kept frozen in the fitting procedure (while their amplitudes are left free to vary) and (bottom) correspond to: photon index $\Gamma=1.2$ for HMXBs (yellow); photon index $\Gamma=1.4$ and cutoff energy $\epsilon_{\mathrm{c}}=7.5 \mathrm{keV}$ for LMXBs (purple); temperature $k T=2 \mathrm{keV}$ and chemical abundance $Z=Z_{\odot}$ for SNRs (cyan). At lower energies, three plasma components (having $k T=0.065,0.45,0.75 \mathrm{keV}$; and $Z=0.1 Z_{\odot}$ ) are required (red, green, and dark blue). Shown are the model fitted to the data (top, photon spectrum), the residuals of the fit (middle), and the unfolded energy spectrum (bottom).

component is flat enough $(\Gamma \sim 1.4$ : Zezas et al. 1998) to be accounted for by HMXBs alone, and hence $f \sim 1$. In conclusion, our additional spectral analysis supports the deduction that SBGs have $f \sim 0.2$, while showing a high scatter in the actual value of this factor.

The possibility that the emission of SBGs may be dominated by a few very luminous point sources (e.g., for M 82 see Kaaret et al. 2001 and Strohmayer \& Mushotzky 2003), and that analyses of spatially integrated SBG spectra may be biased, motivates further attempts to estimate $f$. Information about the relative emission from HMXBs can also be derived from studies on point source populations in nearby galaxies based on the high angular resolution Chandra data. Studies
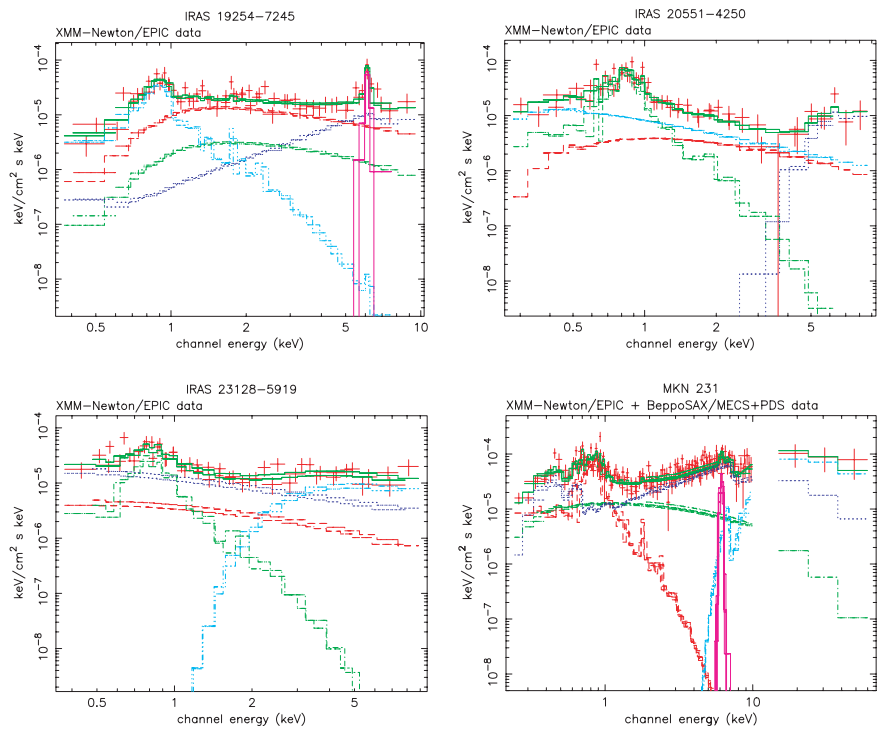

Fig. 3. The energy spectra of the four AGN-contaminated ULIRGs of the Franceschini et al. (2003) sample. The XMM-Newton data for IRAS 19254-7245, IRAS 20551-4250 and IRAS 23128-5919 span the $\sim 0.4-10 \mathrm{keV}$ energy range, while the composite $X M M$-Newton + BeppoSAX data for Mkn 231 span the $0.2-60 \mathrm{keV}$ energy range. The HMXB contributions have been estimated by perturbing the published best-fit spectral models with an additional $\Gamma=1.2 \mathrm{PL}$. Such additional components are reported in the four panels (clockwise from top left) as green, red, green, and red lines.

on individual local galaxies have yielded luminosity functions of X-ray point sources (XPLFs) down to limiting luminosities of $\sim 10^{36-37} \mathrm{erg} \mathrm{s}^{-1}$ (e.g., Kilgard et al. 2002; Colbert et al. 2003; Fabbiano et al. 2001; Zezas et al. 2002, 2003; Roberts et al. 2002a; Soria \& Wu 2002; Bauer et al. 2001; Griffiths et al. 2000; Swartz et al. 2003; Kaaret 2002; Soria et al. 2003; Gao et al. 2003; Terashima \& Wilson 2003; Kong 2003). Such XPLFs can be described as $N(>L) \propto L^{-\alpha}$ with $\alpha \sim 0.5,1,1.5$ for starburst, normal spiral, and elliptical galaxies (and bulges), respectively (Kilgard et al. 2002 and references therein). The difference in XPLFs among galaxy types, which implies that higher-SFR galaxies have relatively more sources (presumably, HMXBs) at high luminosities, is presumably related to the age of the underlying X-ray binary population. A simple birth-death model of X-ray binaries predicts a difference of 1 between the XPLF slope for an equilibrium $\mathrm{XP}$ population and that for an impulsively-formed quietlyageing XP population. However, XP counts do not give clues as to the nature of the XPs. Prestwich et al. (2002) have proposed to classify XPs in external galaxies on the basis of their X-ray colors (defined using Chandra's $0.65,1.5$, and $5 \mathrm{keV}$ bands), finding highly significant differences in the X-ray colors of bulge and disk XPs, i.e. of LMXBs and of HMXBs and SNRs. Colbert et al. (2003) have analyzed 1441 XPs detected in 32 nearby galaxies, concluding that the XP luminosity is well correlated with the K-luminosity (i.e., the stellar mass) and the FIR+UV luminosity (i.e., the $S F R$ ), suggesting that XPs are connected to both the old and young stellar populations. Using their X-ray color diagrams, we find that $\sim 0.2$ of the XPs observed in spiral and interacting galaxies can be 
classified as HMXBs. Correspondingly, a fraction $\sim 0.25$ of the 0.3-8 keV XP luminosity of these galaxies can be attributed to such $\Gamma \sim 1.2$ sources. Because the XP luminosity typically accounts for $\sim 80 \%-90 \%$ of the total luminosity (e.g., Roberts et al. 2002a), we deduce that (putative) HMXBs account for $\sim 20 \%$ of the integrated $0.3-8 \mathrm{keV}$ luminosity. This value is in reasonable agreement with our spectral estimate of $f \sim 0.2$, mostly based on $\mathrm{M} 82^{7}$. We therefore consider $f \sim 0.2$ to be the currently appropriate, representative average value, for the SBG sample.

An analogous correction to the FIR emission, aimed at removing any contribution that is unrelated to ongoing SF activity, is also in order. As discussed in Sect. 2.1, we must subtract the (SB-unrelated) cirrus component from the FIR emission of $\mathrm{SBGs}^{8}$. (The practical impact of this necessary correction is minor (see Fig. 4), and thus has no bearing on any of our results.)

The $S F R$, as measured by $f L_{2-10}$, turns out to be in good agreement with that inferred from $L_{\mathrm{FIR}}$ (see Fig. 4, filled squares $^{9}$ ); the left panel includes the correction for the cirrus component in the FIR emission. To appreciate the effect of the

7 The integrated XP luminosity of SFGs is dominated by ultraluminous X-ray sources (ULXs), i.e. off-nucleus objects with $L \geq$ $10^{39} \mathrm{erg} \mathrm{s}^{-1}$ which is the Eddington luminosity for a $8 M_{\odot}$ black hole (BH) (which is the limiting BH mass obtainable via stellar evolution). The nature of ULXs is still unclear. However, ULXs appear to be related mostly to high-SFR environments (Fabbiano et al. 2001; Lira et al. 2002; Roberts et al. 2002a; Zezas et al. 2002, 2003; Humphrey et al. 2003; Gao et al. 2003; Karet et al. 2001; Strickland et al. 2001): the identified optical counterparts of ULXs are indeed O stars, so suggesting a relation of ULXs with HMXBs (Liu et al. 2002; Roberts et al. 2002b). The spectra of ULXs, reminiscent of those of Galactic BH $\mathrm{X}$-ray binary candidates in high state, are described by a model comprising an accretion disk with inner-edge temperature of $\sim 1.1 \mathrm{keV}$ plus a hard $(\Gamma \sim 1.2)$ PL similar to that observed in HMXBs (Zezas et al. 2002; Foschini et al. 2002; see also Fabbiano \& White 2003). A scenario in which a population of HMXBs, with $\gtrsim 15 M_{\odot}$ donors transferring mass on their thermal timescales (and hence at super-Eddington rates) to $\gtrsim 10 M_{\odot}$ accretors, fits current observations on ULXs (King 2003). Our computation of $f \sim 0.2$, based on the XP colors corresponding to $\Gamma \sim 1.2$, does therefore incorporate the possible presence of ULXs.

${ }^{8}$ Some objects in Table 2 are found at low Galactic latitudes $(|b|<15$ degrees). For these the corrections for foreground Galactic absorption tend to be large and uncertain. An extreme case is IC 342 ( $b=10.58 \mathrm{deg}$ ), for which the $B$-band absorption is estimated to be as large as 3.360 mag (Burstein \& Heiles 1982) or 2.407 mag (Schlegel et al. 1998). As the adopted statistical correction for cirrus emission turns out to be unphysical in this case, we will leave IC 342 out from further analyses involving cirrus-corrected FIR luminosities. No result in this paper will depend on whether this object is included in (or excluded from) the sample. (Note that IC 342 is uncharacteristically underluminous in FIR and soft X-rays compared with hard X-rays, suggesting the presence of a HMXB population typical of a SBG, but with reduced FIR and hot gas emissions, Bauer et al. 2003.)

9 The SBG sample contains one object, Arp 299, which was recently shown to host an AGN (Della Ceca et al. 2002). Remarkably, this object lies in the region populated by the AGN-dominated ULIRGs.
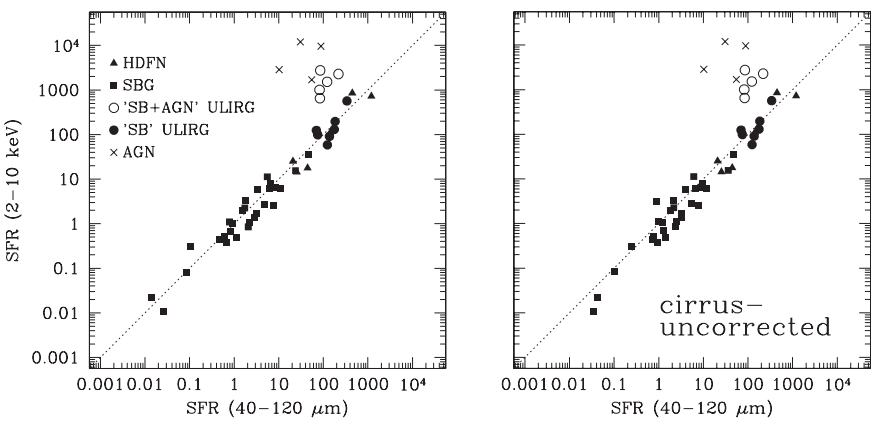

Fig. 4. The $S F R$ estimated from $\mathrm{X}$-ray emission versus that estimated from FIR emission. For all objects it is assumed that the emission in both bands is related to starburst activity. Filled squares and filled dots represent, respectively, SBGs and SB-dominated ULIRGs; empty dots denote SB/AGN-powered ULIRGs, while crosses denote AGN-dominated objects; filled triangles denote HDFN galaxies (for which, due to the lack of FIR measurements, the $S F R$ s have been estimated from radio fluxes). The dotted line, representing the $S F R_{\mathrm{x}}=$ $S F R_{\text {FIR }}$ relation, is only meant to guide the eye. The right panel shows the case where the FIR luminosities of normal and starbursting galaxies are not corrected for cirrus emission.

correction, in the right panel we show the plot without the cirrus correction.

- Hubble Deep Field North Galaxies. In the $S F R-L_{2-10}$ plane the HDFN galaxies overlap (with a wider spread in $S F R$ ) with the SB-dominated ULIRGs (see Fig. 5-left) ${ }^{10}$.

Lacking spectral information, a simple and realistic assumption is that HDFN galaxies - likely "juveniles" - are SF-dominated. Indeed Cohen (2003) finds that, based on the emitted luminosity in the $3727 \AA$ line of [O II], the average $S F R$ is about an order of magnitude higher in HDFN galaxies than in local ones. Furthermore, the age of a galaxy at $z \sim 1, \sim 6 \mathrm{Gyr}$, is not long enough for LMXBs - which have sub-solar mass donors - to form (see Maeder \& Meynet 1989). These considerations suggest that the $2-10 \mathrm{keV}$ emission of HDFN galaxies is due essentially to HMXBs, like in the SB-dominated ULIRGs discussed above. We then set $L_{2-10}^{\mathrm{HMXB}}=L_{2-10}$. The X-ray estimates of the $S F R$ agree comfortably with those inferred from the radio data (see Fig. 4). Therefore, the $S F R-L_{2-10}^{\mathrm{HMXB}}$ relation for HDFN galaxies is consistent with that for SBGs and SB-dominated ULIRGs (see Fig. 5-right).

Analysis of the three samples leads us to the following conclusions:

i) in SB-dominated ULIRGs the $2-10 \mathrm{keV}$ luminosity is a gauge of the $S F R$. This result lends further support to the interpretation of the $\sim 2-10 \mathrm{keV}$ emission of ULIRGs as being (mainly) the integrated emission from HMXBs. In those cases where an AGN clearly shows up (Franceschini et al. 2003) when this component is subtracted out, the residual $2-10 \mathrm{keV}$ luminosities imply $S F R$ values that agree with the FIR-based ones;

${ }^{10}$ Had we mistakenly used the plain $\operatorname{SFR}\left(\geq 5 M_{\odot}\right)$ values resulting from applying Condon's (1992) formula to the radio data - instead of the extrapolated $S F R\left(\geq 0.1 M_{\odot}\right)$ values - the HDFN galaxies would have lined up, and partially overlapped, with the SBGs in the $S F R-L_{2-10}$ plane. 

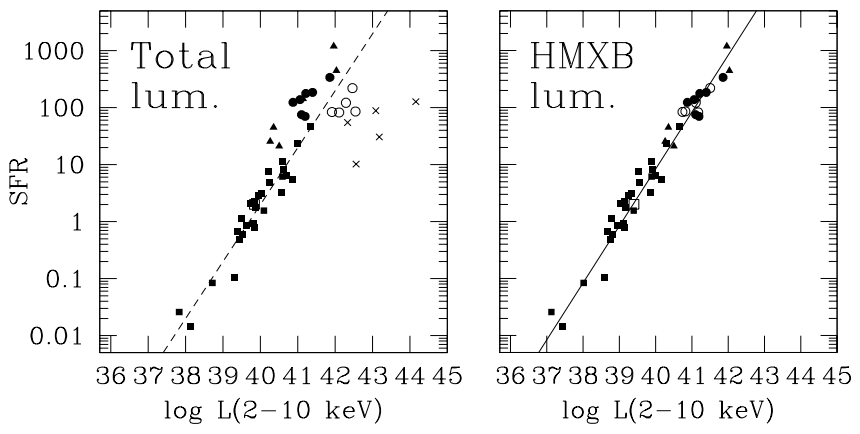

Fig. 5. The $S F R$ versus $2-10 \mathrm{keV}$ luminosity relations, using the total luminosity (left) and the HMXB luminosity (right). The SFR has been computed from the cirrus-subtracted FIR luminosities according to Eq. (1) (except for the HDFN sample (filled triangles) where radio data have been used; only X-ray detections have been used, see Table 3). Symbols are as in Fig. 4; the large empty square represents the Milky Way $\left(S F R \simeq 2 M_{\odot} \mathrm{yr}^{-1}\right.$, see Matteucci $2002 ; L_{2-10} \sim 2 \times 10^{39} \mathrm{erg} \mathrm{s}^{-1}$ and $L_{2-10}^{\mathrm{HMXB}} \sim 4 \times 10^{38} \mathrm{erg} \mathrm{s}^{-1}$, estimated from Iben et al. 1995a,b and consistent with the template spectrum in Fig. 1). The solid line in the right panel shows the linear relation in Eq. (2), whereas the dashed line in the left panel shows the Ranalli et al. (2003) relation $S F R=L_{2-10} /\left(5 \times 10^{39} \mathrm{erg} \mathrm{s}^{-1}\right) M_{\odot} \mathrm{yr}^{-1}$.

i) in local star-forming (i.e., normal and starburst) galaxies only a relatively minor fraction $(f \sim 0.20)$ of the $2-10 \mathrm{keV}$ luminosity is related to the ongoing SF activity. Correcting for this bias results in essentially the same $S F R-L_{2-10}^{\mathrm{HMXB}}$ relation for both ULIRGs and SBGs;

iii) distant $(z \sim 1)$ SFGs have $S F R$ values and $2-10 \mathrm{keV}$ luminosities similar to those of ULIRGs.

Within the uncertainties of the data (most notably those involved in identifying the HMXB contribution in our sample of AGN-contaminated objects) the resulting relation is roughly linear,

$\operatorname{SFR}\left(\geq 0.1 M_{\odot}\right)=\frac{L_{2-10}^{\mathrm{HMXB}}}{10^{39} \mathrm{erg} \mathrm{s}^{-1}} M_{\odot} \mathrm{yr}^{-1}$

with an estimated $20 \%$ statistical error.

It should be remarked that the $S F R$-luminosity relation is not tight if no correction is applied (see Fig. 5-left). Both SBGs and AGN-contaminated ULIRGs are overluminous for their SFR when compared with the SB-dominated ULIRGs and HDFN galaxies. Indeed, SBGs are contaminated by substantial LMXB emission, and in AGN-contaminated ULIRGs the AGN emission is typically brighter than the SB emission. After correcting for both types of excess, no hint of non-linearity appears in the $S F R$-luminosity relation (Fig. 5-right).

\section{Discussion}

Our main suggestion - and the central theme of investigation has been the notion that the $2-10 \mathrm{keV}$ collective emission of HMXBs, $L_{2-10}^{\mathrm{HMXB}}$, is a meaningful gauge of ongoing galactic SFR. As spelled out in Sect. 4, given the universality of the accretion process onto NS/BH in binaries, the linear dependence of $L_{\mathrm{FIR}}$ on the $S F R$, the direct link between the FIR and HMXB emissions, a tight relation is predicted between
$S F R$ and $L_{2-10}^{\mathrm{HMXB}}$. Whether the latter relation is also linear depends largely on whether the stellar mass range and the shape of the IMF are the same in all galaxies, or vary systematically with the $S F R$. In the former case we expect the relation to be linear, whereas if the IMF becomes progressively more top-heavy (i.e., the lower mass cut-off becomes progressively higher) with increasing $S F R$, a non-linear relation is expected.

As we have mentioned, the use of HMXBs as a galactic SFR estimator was already proposed by Grimm et al. (2003) (see also David et al. 1992). Within the general agreement between their background picture and ours, the main result of Grimm et al. is, however, quite different from ours: their resulting relation is non-linear $\left(S F R \propto L^{0.6}\right)$ for small $S F R$ and low values of $L_{2-10}$ (i.e., $S R F \lesssim 4.5 M_{\odot} \mathrm{yr}^{-1}$ and $L_{2-10} \lesssim$ $2.6 \times 10^{40} \mathrm{erg} \mathrm{s}^{-1}$ ), and linear for higher values of the $S F R$ and $L_{2-10}$. Gilfanov et al. (2004) argue that such non-linearity in the low-SFR limit may be caused by non-Gaussianity of the probability distribution of the integral distribution of discrete sources. We can offer no clear explanation for the discrepancy between our result and theirs. One important difference between our approach and that of Grimm et al. (2003) is their a priori minimization of the LMXB contribution because - as they put it - "owing to the absence of optical identifications of a donor star in the X-ray binaries detected by Chandra in other galaxies, ... there is no obvious way to discriminate the contribution of low-mass X-ray binaries”. Grimm et al. argue that, given the long evolution timescales of LMXBs, in a galaxy the LMXB population should be proportional to the galaxy stellar mass, whereas the HMXB population should be proportional to the $S F R$, so that the relative importance of LMXBs should be characterized by the ratio of the stellar mass to the SFR. Using dynamical estimates for the stellar masses of galaxies, and $S F R$ derived from a variety of indicators $(\mathrm{UV}, \mathrm{H} \alpha, \mathrm{FIR}$, radio, that give a wide range of values of the $S F R$ - see their Table 3), Grimm et al. claimed that they selected only galaxies where HMXB emission is expected to exceed LMXB emission by a factor of $\gtrsim 3$. For their sample galaxies, consequently, Grimm et al. supposed that the plain $2-10 \mathrm{keV}$ luminosity is a measure of the collective HMXB emission in that band. In contrast, our procedure involves the identification of the HMXB emission from a given SFG by means of a spectral decomposition of the observed X-ray spectrum.

The need in our approach to single out $L_{2-10}^{\mathrm{HMXB}}$ rather than $L_{2-10}$ is further spurred by the mix of starbursts of widely varying strengths, from low/moderate (local SBGs) to extreme (ULIRGs). While the FIR emission is mostly related to current SF in both cases (see Sect. 2.1), the 2-10 emission is not in the former case but it is in the latter. Had we restricted to the local SBG sample, we would have found $S F R \simeq$ $L_{2-10} /\left(5 \times 10^{39} \mathrm{erg} \mathrm{s}^{-1}\right) M_{\odot} \mathrm{yr}^{-1}$ (see Fig. 5-left), in agreement with Ranalli et al. (2003). In fact, the $2-10 \mathrm{keV}$ emission from local SBGs has approximately a similar mix of SB-related (HMXB) emission and SB-unrelated (LMXB) emission, so that for this sample the primary correlation, $S R F \propto L_{2-10}^{\mathrm{HMXB}}$, propagates into $S R F \propto L_{2-10}$. Some extra scatter plagues the latter relation because the value $f \sim 0.2$ appearing in the equality $L_{2-10}^{\mathrm{HMXB}}=f L_{2-10}$ most likely has some appreciable scatter within the sample. Only when the SB-dominated ULIRGs 
and HDFN galaxies are introduced into the plot and are used as calibrators, does the linearity of the SBG $S F R-L_{2-10}$ relation break down (see Fig. 5-left). The linearity of the relation, extended to the whole luminosity range, is re-established by using the SB-related luminosity $L_{2-10}^{\mathrm{HMXB}}$ (see Fig. 5-right). (Of course, the calibration of our relation is lower than that of Ranalli et al.'s relation by the factor $f=0.2$.)

One key assumption underlying our $S F R-L_{2-10}$ relation is that the FIR luminosities - from which the $S F R$ values are computed - are really due to the SB and are not contaminated by other contributions in any important way. In order to check this assumption, we convert the observed $f_{60} / f_{100}$ ratios to dust temperatures using the table in Helou et al. (1988). The distribution of $60 \mu$-to-100 $\mu$ flux density ratios (see Fig. 6-left) implies a range of estimated dust temperature ${ }^{11} 25 \mathrm{~K} \lesssim T \lesssim 50 \mathrm{~K}$ (if the dust emissivity is proportional to $v^{-1}$ ), the lower bound typically referring to more quiescent (i.e., "normal") galaxies (e.g., NGC 628) and the upper bound to more actively star forming objects (e.g., the well-known SB-dominated galaxy Arp 220; Silva et al. 1998). This range of values seems realistic for the samples of SFGs considered in this paper, thus confirming the soundness of the assumption that our FIR luminosities are not significantly contaminated by emission unrelated to ongoing SF activity, and verifying earlier suggestions that galaxies with higher $S F R$ s have higher dust temperatures (e.g., David et al. 1992; but there may be exceptions to this trend, see Fig. 6left). Notice also that the ratio of the two SB-related luminosities, $L_{2-10}^{\mathrm{HMXB}} / L_{\mathrm{FIR}}$, remains approximately constant when moving from lower temperatures (normal and starburst galaxies) to higher temperatures (ULIRGs), $\log \left(L_{2-10} / L_{\mathrm{FIR}}\right) \simeq-4.3 \pm 0.3$ (see Fig. 6-right). In particular, note that, when properly accounting for LMXB and AGN emission, SBGs and ULIRGs imply an X-to-FIR ratio consistent with that for the pure SBs arguably represented by the AGN-free ULIRGs.

In the simple scenario, in which the formation of OB stars and of accreting NSs/BHs during episodes of active SF activity only depends on local conditions, the same FIR-X-ray emission relation is expected to hold for local $(z \lesssim 0.1)$ and distant $(z \sim 1)$ star-forming galaxies. Indeed, the sample of $z \sim 1$ HDFN galaxies we have used does follow the $S F R-L_{\mathrm{x}}$ relation established in local galaxies. It should be emphasized that distant galaxies are in a substantially active SF phase: their 2-10 keV emission must then be HMXB-dominated, if no central AGN is contaminating the emission. If an independent estimate of the $S F R$ is also available (e.g., from deep radio observations), the location of the galaxy in the $S F R-L_{2-10}$ plane can be used to evaluate any excess X-ray luminosity which, if detected, would presumably imply the presence of an AGN. For example, if according to Eq. (2) a distant galaxy were very overluminous for its $S F R$ (e.g., if $L_{2-10} \sim 10^{43} \mathrm{erg} \mathrm{s}^{-1}$ for

\footnotetext{
11 Because galaxies have multiple emission components with widely varying parameters, the ratio $f_{60} / f_{100}$ cannot be expected to yield a precise temperature. The uncertainty affecting dust temperature estimates is largely intrinsic, and can be traced back to substantial uncertainties that are essentially of astrophysical origin (e.g., the frequency dependence of the dust emissivity, e.g., Helou et al. 1988).
}
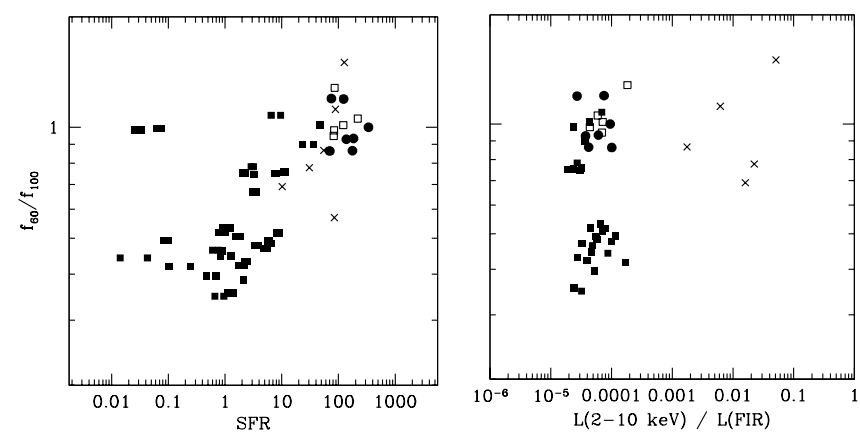

Fig. 6. The distribution of $60 \mu$-to- $100 \mu$ flux density ratios as a function of the SFR (left) and of the SB $2-10 \mathrm{keV}$ to FIR luminosity ratio (right). Because $f_{60} / f_{100}$ is an approximate measure of the temperature, these plots show that: $(i)$ in spite of a relatively large scatter, a $S F R$-temperature correlation is present in the data, whereby galaxies with higher (lower) $S F R$ tend to have higher (lower) dust temperature, $T \sim 50(25) \mathrm{K}$; and (ii) the ratio of HMXB 2-10 keV luminosity to dust FIR emission remains roughly constant irrespective of the dust temperature. Symbols are as in Fig. 4.
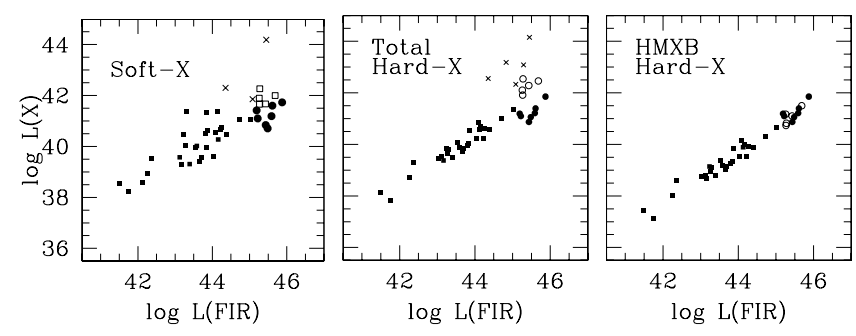

Fig. 7. The X-ray-FIR luminosity relation for soft (mostly $0.5-2 \mathrm{keV}$ (left)) and hard X-rays (2-10 keV: total emission (center), and HMXB emission $(r i g h t)$ ). In all panels the FIR luminosities of the SBG sample have been corrected for cirrus emission. Symbols are as in Fig. 4.

$S F R \sim 100 M_{\odot} \mathrm{yr}^{-1}$ ), we might suspect a significant AGN contribution to the observed 2-10 keV emission (evolutionary considerations rule out significant LMXB emission). This method, based on joint X-ray and (say) radio observations, could be a very efficient tool to detect AGNs that are buried deeply (i.e., absorbed through $N_{\mathrm{H}}>10^{23} \mathrm{~cm}^{-2}$ ) in the nuclei of distant SFGs and are not detectable in other bands (e.g., the exemplary cases of NGC 6240 Vignati et al. 1999, NGC 4945 Guainazzi et al. 2000, Arp 299 Della Ceca et al. 2000). We therefore caution against using $L_{2-10}$ as a gauge of the $S F R$.

Finally, from Fig. 7-(left) we see that the emission of SFGs at $\sim 1 \mathrm{keV}$ is also an indicator of the $S F R$, although the scatter of the $S F R-L_{\mathrm{x}}$ relation is higher in the soft band than in the hard band (see Fig. 7, where the equivalent $L_{\mathrm{FIR}}-L_{\mathrm{x}}$ relation is shown). Part of the scatter seen in Fig. 7-(left) is due to observational errors - such as uncertainties in determining the absorption and metallicity of the soft thermal plasma, or occasional differences in the definitions of the soft spectral bands but most of it is probably intrinsic. In fact, the soft component which is systematically observed in the spectra of SFGs is interpreted as sub-keV thermal emission originating from SN-powered outgoing galactic winds (e.g., Dahlem et al. 1998; Franceschini et al. 2003). Spatially resolved data (Strickland et al. 2000) and simulations (Strickland \& Stevens 2000) have shown that this emission occurs at the boundary between the 
hot, tenuous wind fluid proper and the cool, denser ISM where conditions are optimal for thermal emission of X-rays. This implies that only a small fraction of the wind mass is involved in $\mathrm{X}$-ray emission, and that local conditions (i.e., density, clumpiness, and chemical composition of the ISM) crucially determine the level of this emission. The small mass fraction of thermally X-ray emitting galactic gas is subject to a large scatter among galaxies. These considerations suggest that most of the scatter observed in Fig. 7-(left) is probably intrinsic. Indeed, the pure thermal emission does not generally correlate with $L_{\text {FIR }}$ (see Fig. 6c of Franceschini et al. 2003), implying that galacticwind emission is not a SFR indicator (in spite of winds being an immediate SB outcome). Then, we suggest that the observed $L_{0.5-2}-L_{\text {FIR }}$ correlation is not primary and mainly due to SB-powered galactic winds in the SFGs, but that it is probably induced by the portion of the hard spectral component of SFGs showing up in the $0.5-2 \mathrm{keV}$ band. Notice how the $L_{\mathrm{x}}-L_{\mathrm{FIR}}$ relation improves when going from left to right in Fig. 7, i.e. when the reference X-ray luminosity changes from "total soft" through "total hard" to "HMXB hard".

\section{Conclusion}

Current limited ability to spectrally decompose the $2-10 \mathrm{keV}$ emission of SFGs is nonetheless indicative of the feasibility of using the main HMXB component as an estimator of the galactic $S F R$. The total $2-10 \mathrm{keV}$ luminosity is not a proper $S F R$ indicator since for low values of the $S F R$ - in normal and moderately-starbursting galaxies - it is substantially affected by the emission of LMXBs, while for high values of the SFR in ULIRGs - it usually includes significant emission from obscured AGNs.

The use of an additional sample of distant galaxies from the Hubble Deep Field North, for whom there is no X-ray spectral information, but whose $S F R$ can be estimated from deep radio data, led us to to conjecture that the $S F R-L_{\mathrm{x}}$ relation that we deduced for local galaxies might also hold for distant, $z \sim 1$, galaxies, if the 2-10 keV emission of HDFN galaxies is dominated by HMXBs.

The $S F R-L_{2-10}^{\mathrm{HMXB}}$ relation may be universal across galaxy types and redshifts. This may not be so surprising, if our understanding of the processes involved is basically correct. However, its linearity and calibration, both in the local and in the distant Universe, are major issues that remain to be settled. Resolved high-energy long-exposure $X M M$-Newton spectra will be needed to afford detailed modelling of the integrated emission, while high-spatial-resolution Chandra imaging will be needed to construct the luminosity functions of HMXBs. Achieving mutually consistent results from the spectral decomposition and from the integration of the HMXB luminosity function would lead to a definite determination of $L_{2-10}^{\mathrm{HMXB}}$.

Once the universality of the $S F R-L_{2-10}^{\mathrm{HMXB}}$ relation has been cleary established, it will be possible to use the relation as a tool to unveil the presence of deeply absorbed AGNs lurking in the centers of distant galaxies. If, for its (independently estimated) $S F R$, the $2-10 \mathrm{keV}$ luminosity of a distant galaxy will be measured to be in excess of that predicted based on the
$S F R-L_{\mathrm{x}}$ relation, that galaxy would be suspected to harbor a strongly absorbed AGN.

Finally, we stress again that in order to build a physically sound X-ray SFR indicator, we should use, in principle, a sample of star-forming galaxies whose X-ray spectral properties are well known, and for which the $S F R$ estimates are robust. Extracting the HMXB 2-10 keV emission is clearly one crucial step. Ideally, high-quality spectra should be available and analyzed for each object in the sample. We unfortunately do not yet have such data. At moderate- and low-SFR regimes we relied on estimates gained from analyzing data for few objects (M 82, M 83, and NGC 253; plus educated guesses from NGC 2146, NGC 2903, NGC 3310, NGC 3256), which we then assumed would hold for the whole sample of normal and starburst galaxies. In the high-SFR regime we do have suitable spectral analyses for the purely SB-powered ULIRGs, but we had to resort again to estimates in the case of AGN-contaminated ULIRGs. For the sample of distant HDFN star-forming galaxies we could only make (educated) assumptions. The situation is clearly far from ideal. Therefore our results, although promising are clearly preliminary. With the ongoing acquisition of spatially resolved Chandra data and spectrally resolved XMM-Newton data, it should be possible to observe galactic regions that are clearly recognized sites of ongoing SF, in particular the actively star-forming central regions of starburst galaxies. These smaller starbursts, along with those ULIRGs which are not contaminated by AGNs and can be considered as giant starbursts, could yield a well defined $S F R-L_{\mathrm{x}}$ relation. So far we have been able to suggest a continuity of SF properties moving from normal through starburst to ultra-luminous IR galaxies (quantified by $L_{2-10}^{\mathrm{HMXB}} / L_{\mathrm{FIR}} \simeq 5 \times 10^{-5}$ over about 5 decades in X-ray luminosity). But the $S F R-L_{\mathrm{x}}$ relation we have obtained is still affected by a fairly large scatter, which would currently limit its applicability even in the case of an object with a well resolved HMXB emission. The picture is emerging, the details are still missing.

Acknowledgements. This research has made use of the NASA/IPAC Extragalactic Database (NED) which is operated by the Jet Propulsion Laboratory, California Institute of Technology, under contract with the National Aeronautics and Space Administration. Partial financial support to this project was provided by MIUR through contract 2001028932002 and by ASI through contracts $\mathrm{I} / \mathrm{R} / 037 / 01$ and $\mathrm{I} / \mathrm{R} / 00206202$. We acknowledge the HEASARC for providing the $A S C A$ archival data. We thank an anonymous referee for a very careful and critical reading of the manuscript.

\section{References}

Alexander, D. M., Aussel, H., Bauer, F. E., et al. 2002, ApJ, 568, L85 Bauer, F. E., Alexander, D. M., Brandt, W. N., et al. 2002, AJ, 124, 2351

Bauer, F. E., Brandt, W. N., \& Lehmer, B. 2003, AJ, 126, 2797

Bauer, F. E., Brandt, W. N., Sambruna, R. M., et al. 2001, ApJ, 122, 182

Bernlöhr, K. 1993, A\&A, 270, 20

Blandford, R. D., \& Ostriker, J. P. 1980, ApJ, 237, 793

Blustin, A. J., Branduardi-Raymont, G., Behar, E., et al. 2003, A\&A, 403,481 
Braito, V., Franceschini, A., Della Ceca, R., et al. 2003, A\&A, 398, 107

Braito, V., Della Ceca, R., Piconcelli, E., et al. 2004, A\&A, in press

Brandt, W. N., Fabian, A. C., Takahashi, K., et al. 1997, MNRAS, 290, 617

Burstein, D., \& Heiles, C. 1982, AJ, 87, 1165

Cappi, M., Persic, M., Bassani, L., et al. 1999, A\&A, 350, 777

Christian, D. J., \& Swank, J. H. 1997, ApJS, 109, 177

Cohen, J. G. 2003, ApJ, 598, 288

Colbert, E. J. M., Heckman, T. M., Ptak, A. F., \& Strickland, D. K. 2003, ApJ, in press [arXiv: astro-ph/0305476]

Condon, J. J. 1992, ARA\&A, 30, 575

Dahlem, M., Weaver, K. A., \& Heckman, T. M. 1998, ApJS, 118, 401

Dahlem, M., Parmar, A., Oosterbroek, T., et al. 2000, ApJ, 538, 555

David, L. P., Jones, C., \& Forman, W. 1992, ApJ, 388, 82

Della Ceca, R., Griffiths, R. E., Heckman, T. M., \& MacKenty, J. W. 1996, ApJ, 469, 662

Della Ceca, R., Griffiths, R. E., \& Heckman, T. M. 1997, ApJ, 485, 581

Della Ceca, R., Griffiths, R. E., Heckman, T. M., et al. 1999, ApJ, 514, 772

Della Ceca, R., Pellegrini, S., Bassani, L., et al. 2001, A\&A, 375, 781

Della Ceca, R., Ballo, L., Tavecchio, F., et al. 2002, ApJ, 581, L9

de Naray, P. J., Brandt, W. N., Halpern, J. P., \& Iwasawa, K. 2000, AJ, 119,612

Devereux, N. A., \& Eales, S. A. 1989, ApJ, 340, 708

Devereux, N. A., \& Young, J. S. 1991, ApJ, 371, 515

de Vaucouleurs, G., de Vaucouleurs, A., Corwin, H. G. Jr., et al. 1991, Third Reference Catalogue of Bright Galaxies (New York: Springer Verlag)

Doane, J. S., \& Mathews, W. G. 1993, ApJ, 419, 573

Done, C., Madejski, G. M., Zycki, P. T., \& Greenhill, L. J. 2003, ApJ, 588, 763

Engelbracht, C. W. 1997, Ph.D. Thesis, Univ. of Arizona

Fabbiano, G., \& White, N. E. 2003, in Compact Stellar X-Ray Sources, ed. W. Lewin, \& M. van der Klis (Cambridge University Press) [arXiv: astro-ph/0307077]

Fabbiano, G., Zezas, A., \& Murray, S. S. 2001, ApJ, 554, 1035

Foschini, L., Di Cocco, G., Ho, L. C., et al. 2002, A\&A, 392, 817

Franceschini, A., Braito, V., Persic, M., et al. 2003, MNRAS, 343, 1181

Fullmer, L., \& Lonsdale, C. 1989, Cataloged Galaxies and Quasars Observed in the IRAS survey: version 2 (Pasadena: Jet Propulsion $\mathrm{Lab}$ )

Gao, Y., Wang, Q. D., Appleton, P. N., \& Lucas, R. A. 2003, ApJ, 596, L171

Garrett, M. A., de Bruyn, A. G., Giroletti, M., et al. 2000, A\&A, 361, L41

Genzel, R., Lutz, D., Sturm, E., et al. 1998, ApJ, 498, 579

Gilfanov, M., Grimm, H.-J., \& Sunyaev, R. 2004, MNRAS, 347, L57

Griffiths, R. E., Ptak, A., Feigelson, E. D., et al. 2000, Science, 250, 1325

Grimm, H.-J., Gilfanov, M., \& Sunyaev, R. 2003, MNRAS, 339, 793

Guainazzi, M., Matsuoka, M., Piro, L., et al. 1994, A\&A, 436, L35

Guainazzi, M., Matt, G., Brandt, W. N., et al. 2000, A\&A, 356, 463

Helou, G., Khan, I. R., Malek, L., \& Boehmer, L. 1988, ApJS, 68, 151

Helou, G., Soifer, B. T., \& Rowan-Robinson, M. 1985, ApJ, 298, L7

Hickson, P., Menon, K., Palumbo, G., \& Persic, M. 1989, ApJ, 341, 679

Holt, S. S., Schlegel, E. M., Hwang, U., \& Petre, R. 2003, ApJ, 588, 792

Hornschemeier, A. E., Brandt, W. N., Garmire, G. P., et al. 2001, ApJ, 554,742
Hornschemeier, A. E., Bauer, F. E., Alexander, D. M., et al. 2003, AJ, 126,575

Humphrey, P. J., Fabbiano, G., Elvis, M., et al. 2003, MNRAS, 344, 134

Hunter, D. A., Gillett, V. C., Gallagher III, J. S., et al. 1986, ApJ, 303, 171

Iben, I. Jr., Tutukov, A. V., \& Yungelson, L. R. 1995a, ApJS, 100, 217

Iben, I. Jr., Tutukov, A. V., \& Yungelson, L. R. 1995b, ApJS, 100, 233

Inoue, A. K., Hirashita, H., \& Kamaya, H. 2000, PASJ, 52, 539

Iwasawa, K. 1999, MNRAS, 302, 96

Iwasawa, K., \& Comastri, A. 1998, MNRAS, 297, 1219

Iwasawa, K., Koyama, K., Awaki, H., et al. 1993, ApJ, 409, 155

Iwasawa, K., Matt, G., Guainazzi, M., \& Fabian, A. C. 2001, MNRAS, 326,894

Kaaret, P. 2002, ApJ, 578, 114

Kaaret, P., Prestwich, A. H., Zezas, A., et al. 2001, MNRAS, 321, L29

Kennicutt, R. C., Jr. 1998, ApJ, 498, 541

Kilgard, R. E., Kaaret, P., Krauss, M. I., et al. 2002, ApJ, 573, 138

King, A. R. 2003, MNRAS, accepted [arXiv: astro-ph/0309450]

Kong, A. K. H. 2003, MNRAS, 346, 265

Leitherer, C., \& Heckman, T. M. 1995, ApJS, 96, 9

Lehnert, M., \& Heckman, T. 1996, ApJ, 472, 546

Lira, P., Ward, M., Zezas, A., et al. 2002, MNRAS, 330, 259

Liu, J.-F., Bregman, J. N., \& Seitzer, P. 2002, ApJ, 580, L31

Lonsdale Persson, C. J., \& Helou, G. 1987, ApJ, 314, 513

Lutz, D., Veilleux, S., \& Genzel, R. 1999, ApJ, 517, L13

Maccacaro, T., \& Perola, G. C. 1981, ApJ, 246, L11

Madau, P., Ferguson, H. C., Dickinson, M. E., et al. 1996, MNRAS, 283,1388

Madejski, G., Zycki, P., Done, C., et al. 2000, ApJ, 535, L87

Maeder, A., \& Meynet, G. 1989, A\&A, 210, 155

Martin, C. L., \& Kennicutt, R. C. 1995, ApJ, 447, 171

Matteucci, F. 2002, lecture given at the XIII Canary Islands Winter School of Astrophysics Cosmochemistry: The Melting Pot of Elements [arXiv: astro-ph/0203340]

Meurer, G. R., Heckman, T. M., Lehnert, M. D., et al. 1997, AJ, 114, 54

Mizuno, T., Ohbayashi, H., Iyomoto, N., \& Makishima, K. 1998, in The Hot Universe, ed. K. Koyama et al., IAU Symp., 188, 284

Moran, E. C., Lehnert, M. D., \& Helfand, D. J. 1999, ApJ, 526, 649

Nandra, K., Le, T., George, I. M., et al. 2000, ApJ, 544, 734

Okada, K., Mitsuda, K., \& Dotani, T. 1997, PASJ, 49, 653

Perez-Olea, D. E., \& Colina, L. 1996, ApJ, 468, 191

Persic, M., \& Rephaeli, Y. 2002, A\&A, 382, 843

Persic, M., \& Rephaeli, Y. 2003, A\&A, 399, 9

Persic, M., Cappi, M., Rephaeli, Y., et al. 2003, A\&A, submitted

Prestwich, A. H., Irwin, J. A., Kilgard, R. E., et al. 2002, ApJ, in press [arXiv: astro-ph/0206127]

Ptak, A. F., Serlemitsos, P., Yaqoob, T., \& Mushotzky, R. 1997, AJ, 113,1286

Ptak, A., Heckman, T., Levenson, N. A., et al. 2003, 592, 782

Ranalli, P., Comastri, A., \& Setti, G. 2003, A\&A, 399, 39

Raymond, J. C., \& Smith, B. W. 1977, ApJS, 35, 419

Rephaeli, Y. 1979, ApJ, 227, 364

Rephaeli, Y., \& Gruber, D. E. 2002, A\&A, 389, 2002

Rephaeli, Y., Gruber, D., Persic, M., \& McDonald, D. 1991, ApJ, 380, L59

Richards, E. A. 2000, ApJ, 533, 611

Rieke, G. H., Loken, K., Rieke, M. J., \& Tamblyn, P. 1993, ApJ, 412, 99

Roberts, T. P., Goad, M. R., Ward, M. J., et al. 2002b, in New Visions of the X-ray Universe in the XMM-Newton and Chandra Era [arXiv:astro-ph/0202017] 
Roberts, T. P., Warwick, R. S., Ward, M. J., \& Murray, S. S. 2002a, MNRAS, 337, 677

Salpeter, E. E. 1955, ApJ, 121, 161

Sanders, D. B., Mazzarella, J. M., Kim, D.-C., et al. 2003, AJ, in press [arXiv: astro-ph/0306263]

Sansom, A. E., Dotani, T., Okada, K., et al. 1996, MNRAS, 281, 48

Schlegel, D., Finkbeirer, D. P., \& Davis, M. 1998, ApJ, 500, 525

Schurch, N. J., Roberts, T. P., \& Warwick, R. S. 2002, MNRAS, 335, 241

Severgnini, P., Risaliti, G., Marconi, A., et al. 2001, A\&A, 368, 44

Schulz, H., Komossa, S., Berghoefer, Th. W., \& Boer, B. 1998, A\&A, 330,823

Silva, L., Granato, G. L., Bressan, A., \& Danese, L. 1998, ApJ, 509, 103

Soria, R., Pian, E., \& Mazzali, P. A. 2003, A\&A, in press [arXiv: astro-ph/0304526]

Soria, R., \& Wu, K. 2002, A\&A, 384, 99

Strickland, D. K., Colbert, E. J. M., Heckman, T. M., et al. 2001, ApJ, 560, 707

Strickland, D. K., Heckman, T. M., Weaver, K. A., \& Dahlem, M. 2000, AJ, 120, 2965

Strickland, D. K., \& Stevens, I. R. 2000, MNRAS, 314, 511
Strohmayer, T. E., \& Mushotzky, R. F. 2003, ApJ, 586, L61

Suchov, A. A., Balsara, D. S., Heckman, T. M., \& Leitherner, C. 1994, ApJ, 430, 511

Swartz, D. A., Ghosh, K. K., McCollough, M. L., et al. 2003, ApJS, 144, 213

Terashima, Y., \& Wilson, A. S. 2003, ApJ, accepted [arXiv: astro-ph/0305563]

Thompson, R. I., Weymann, R. J., \& Storrie-Lombardi, L. J. 2001, ApJ, 546, 694

Tully, R. B. 1988, Nearby Galaxies Catalog (Cambridge: Cambridge Univ. Press)

Veilleux, S., Kim, D.-C., \& Sanders, D. B. 1999, ApJ, 522, 139

Vignati, P., Molendi, S., Matt, G., et al. 1999, A\&A, 349, L57

Walterbos, R. A. M., \& Greenawalt, B. 1996, ApJ, 460, 696

Xia, X. Y., Xue, S. J., Mao, S., et al. 2002, ApJ, 564, 196

Zezas, A., Fabbiano, G., Rots, A. H., \& Murray, S. S. 2002, ApJ, 577, 710

Zezas, A. L., Georgantopoulos, I., \& Ward, M. J. 1998, MNRAS, 301, 915

Zezas, A., Ward, M. J., \& Murray, S. S. 2003, ApJ, 594, L31

White, N. E., Swank, J. H., \& Holt, S. S. 1983, ApJ, 270, 711 
M. Persic et al.: 2-10 keV HMXB luminosity as galactic SFR indicator, Online Material p 1

\section{Online Material}




\section{Appendix A}

In this Appendix we describe the reduction and analysis of the ASCA and BeppoSAX data for the nearby starburst galaxy M 82.

\section{A.1 Data reduction}

ASCA data. The available archival spectral data for the galaxies M 82, M 83, NGC 253, NGC 2146, NGC 2903, NGC 3256, NGC 3310 were retrieved from the HEASARC for analysis. Net spectra for entire observations, typically of duration 20-30 ks, were obtained, along with response matrices. Provided by the project were also the background spectra of the GIS detectors. The SIS backgrounds have been computed by accumulating cleaned counts arriving in a wide annulus surrounding (but excluding) the source into background spectra. Using software provided by the project we then generated response matrices for these accumulations ${ }^{12}$. Spectral fitting was performed simultaneously on the two SIS and two GIS detectors, with a bandpass of $0.4-8 \mathrm{keV}$ for SIS and $0.8-8 \mathrm{keV}$ for GIS. We made sure we could closely reproduce the data and the results presented for the galaxies in the list by the original authors (M 82, NGC 253: Ptak et al. 1997; M 83: Okada et al. 1997; NGC 2146: Della Ceca et al. 1999; NGC 2903: Mizuno et al. 1998; NGC 3256: Moran et al. 1999; NGC 3310: Zezas et al. 1998).

BeppoSAX data. The reduction of the BeppoSAX data for M 82 is described in detail in Cappi et al. (1999). Here let it suffice to say that our analysis is restricted to the $0.1-4.5 \mathrm{keV}$ and 1.5-10 keV energy bands of the LECS and MECS instruments, where the response matrices are best calibrated. The extraction region, $4^{\prime}$, has been chosen in order to maximize the $\mathrm{S} / \mathrm{N}$ ratio. Standard blank-sky files (provided by the BeppoSAX Data Center) were used for background subtraction: the background turned out to provide $\sim 7 \%$ of the total counts at $6 \mathrm{keV}$. The detection of M 82 in the PDS data (nominal band: $13-300 \mathrm{keV}$ ) is statistically significant up to $\sim 30 \mathrm{keV}$. However, some degree of contamination from M 81 is possible due to the latter's higher flux (by a factor 2.5 ) and angular proximity (which ensures that about half of its flux is collected). On the other hand, the spectral slopes of M 81 and M 82 in the PDS band do differ from each other, suggesting a tighter constraint on the contamination from $M$ 81. To add uncertainty to the issue, it should be recalled that M 81 is known to have varied by a factor $\gtrsim 4$ on a timescale of months/years. These considerations led Cappi et al. (1999) to a conservatively assume that the PDS data provided only upper limits. In our analysis here, although we used all (including PDS) data, we made sure that the main result of our analysis (i.e., the estimate of the fractional $2-10 \mathrm{keV} \mathrm{lu}-$ minosity arising from the HMXB population) is substantially solid against including or rejecting the PDS data.

\footnotetext{
${ }^{12}$ Note that the derived spectral parameters are sensitive to the background subtraction. Without a proper background subtraction, the hard $\mathrm{X}$-ray spectrum of the source will appear systematically flatter, and the HMXB contribution will be overestimated.
}

\section{A.2 Data analysis}

Following Persic \& Rephaeli (2002), the model spectrum we have used to analyze the M 82 emission comprises a thermal plasma plus the stellar-end product populations of SNRs, HMXBs, and LMXBs.

As for the thermal plasma component, we found that the details of the fit at $\epsilon \lesssim 1 \mathrm{keV}$ do not appreciably affect the results of the fit (i.e., the amplitudes of the stellar endproducts components) at $\epsilon \gtrsim 2 \mathrm{keV}$, and vice versa. However, an accurate low-energy fit was required in order to give an acceptable value of $\chi_{v}^{2}$. So we used a multi-phase thermal plasma to fit the $<1 \mathrm{keV}$ data, allowing both temperature and amplitude to vary, but forcing one same chemical abundance for all the phases. In fact, we do have theoretical insights (e.g., Suchkov et al. 1994) and some observational evidence (e.g., Dahlem et al. 1998; Strickland et al. 2000) on the existence of multi-phase $\mathrm{X}$-ray emitting thermal plasmas in starburst environments, but no well-constrained ranges for the temperature and the chemical composition are available other than the rather generic (and expected, based on energy considerations) hint that the temperatures should be $k T \lesssim 1 \mathrm{keV}$, and the chemical abundances should be $Z \lesssim Z_{\odot}$. Specifically, we found that three plasma phases, described by Raymond-Smith (1977) models having temperatures $k T=0.065,0.45,0.75 \mathrm{keV}$ and chemical abundance $Z=0.1 Z_{\odot}$, provide an acceptable fit to the low-energy data. The derived temperatures are within the range yielded for the X-ray emitting plasma by numerical simulations of the interaction between SN-driven winds and the ambient ISM in starbursts (Stickland \& Stevens 2000). These parameter values were determined from an exploration of the parameter space $0 \leq k T_{1} \leq k T_{2} \leq k T_{3} \leq 1$, with the constraint that $Z_{1} \equiv Z_{2} \equiv Z_{3}$. Once these parameters were determined, they were kept frozen (in the best-fit procedure) while the corresponding amplitudes $A_{1}, A_{2}$ and $A_{3}$ were left free to vary. As concerns the stellar end-products, we assumed the average spectral shapes observed for the corresponding Galactic populations to hold also in the case of M 82.

The results of the fitting, for the separate as well as joint ASCA and BeppoSAX data sets, are shown in Table A.1. Within the errors, the results of the separate fits and of the joint fit are mutually consistent. 
M. Persic et al.: 2-10 keV HMXB luminosity as galactic SFR indicator, Online Material p 3

Table A.1. Spectral fitting results for $\mathrm{M} 82^{a}$.

\begin{tabular}{|c|c|c|c|c|c|c|c|c|c|}
\hline & & Wind $^{b}$ & & Abs. ${ }^{c}$ & $\mathrm{SNR}^{d}$ & $\mathrm{HMXB}^{e}$ & $\operatorname{LMXB}^{f}$ & & $\mathrm{Fit}^{g}$ \\
\hline & $A_{1}$ & $A_{2}$ & $A_{3}$ & $N_{\mathrm{HI}}$ & $A_{4}$ & $A_{5}$ & $A_{6}$ & $\chi_{v}^{2}$ & d.o.f. \\
\hline \multicolumn{10}{|l|}{ SAX } \\
\hline & $11.07_{-2.70}^{+2.70}$ & $2.11_{-0.73}^{+0.73} \mathrm{E}-2$ & $3.60_{-0.59}^{+0.59} \mathrm{E}-2$ & $1.20_{-0.16}^{+0.16}$ & $6.00_{-2.32}^{+2.32} \mathrm{E}-3$ & $5.04_{-2.46}^{+2.46} \mathrm{E}-4$ & $7.52_{-0.61}^{+0.61} \mathrm{E}-3$ & 1.22 & 139 \\
\hline \multicolumn{10}{|l|}{ ASCA } \\
\hline & $7.64_{-1.06}^{+1.06}$ & $3.43_{-0.38}^{+0.38} \mathrm{E}-2$ & $3.89_{-0.28}^{+0.28} \mathrm{E}-2$ & $1.27_{-0.07}^{+0.07}$ & $9.04_{-2.13}^{+2.13} \mathrm{E}-3$ & $7.74_{-4.16}^{+4.16} \mathrm{E}-4$ & $11.01_{-0.78}^{+0.78} \mathrm{E}-3$ & 1.41 & 969 \\
\hline \multicolumn{10}{|l|}{$\begin{array}{l}\text { SAX+ } \\
\text { ASCA }\end{array}$} \\
\hline & $8.25_{-1.01}^{+1.01}$ & $3.51_{-0.35}^{+0.35} \mathrm{E}-2$ & $3.95_{-0.26}^{+0.26} \mathrm{E}-2$ & $1.23_{-0.06}^{+0.06}$ & $9.23_{-1.64}^{+1.64} \mathrm{E}-3$ & $6.49_{-0.03}^{+0.03} \mathrm{E}-4$ & $11.05_{-0.52}^{+0.52} \mathrm{E}-3$ & 1.42 & 1114 \\
\hline
\end{tabular}

${ }^{a}$ The quoted errors represent the estimated $1 \sigma$ confidence intervals, and are calculated by the X-ray spectral-fitting program XSPEC from the derivatives of the fit statistic with respect to the model parameters.

${ }^{b}$ Raymond-Smith (1977) plasma models with $k T_{1}=0.065 \mathrm{keV}, k T_{2}=0.45 \mathrm{keV}, k T_{3}=0.75 \mathrm{keV}$, and $Z_{1}=Z_{2}=Z_{3}=0.1 Z_{\odot}$. The amplitude of the RS plasma model is defined as $A \equiv 10^{-14} / 4 \pi\left((1+z) D_{\mathrm{A}}\right)^{2} \int n_{\mathrm{e}} n_{\mathrm{H}}$, where $D_{\mathrm{A}}$ is the angular size distance to the source (expressed in cm), $n_{\mathrm{e}}$ and $n_{\mathrm{H}}$ are the electron and hydrogen densities (in $\mathrm{cm}^{-3}$ ).

${ }^{c} \mathrm{HI}$ column density, in excess of the foreground Galactic value, leading to photoelectric absorption of emission: units are $10^{22} \mathrm{~cm}^{-2}$.

${ }^{d}$ RS plasma model with $k T=2$ and $Z=Z_{\odot}$.

${ }^{e}$ Power-law model of the form $A(\epsilon)=A_{5}(\epsilon / 1 \mathrm{keV})^{-\Gamma}$ where $A_{5}$ is the amplitude at $1 \mathrm{keV}$ (expressed in photons keV $\mathrm{km}^{-2} \mathrm{~s}^{-1}$ ) and $\Gamma=1.2$ is the photon index.

${ }^{f}$ Cutoff power-law model of the form $A(\epsilon)=A_{6}(\epsilon / 1 \mathrm{keV})^{-\Gamma} \mathrm{e}^{-\epsilon / \epsilon_{\mathrm{c}}}$, where $A_{6}$ is the amplitude at $1 \mathrm{keV}\left(\right.$ expressed in photons keV $\left.\mathrm{ke}^{-1} \mathrm{~cm}^{-2} \mathrm{~s}^{-1}\right)$, $\alpha=1.4$ is the photon index, and $\epsilon_{\mathrm{c}}=7.5 \mathrm{keV}$ is the cutoff energy of the exponential cutoff (in $\mathrm{keV}$ ).

${ }^{g}$ All model spectra are absorbed through a foreground Galactic HI column density $N_{\mathrm{HI}}=0.427 \times 10^{22} \mathrm{~cm}^{-2}$. 